\title{
Zebrafish (Danio rerio) as an ecotoxicological model for Nanomaterial induced toxicity profiling
}

Suresh K Verma ${ }^{1 \S *}$, Aditya Nandi ${ }^{1 \S}$, Adrija Sinha ${ }^{1}$, Paritosh Patel ${ }^{1}$, Ealisha Jha ${ }^{1}$, Swabhiman Mohanty $^{1}$, Pritam Kumar Panda ${ }^{1,2}$, Rajeev Ahuja ${ }^{2,4}$, Yogendra Kumar Mishra ${ }^{3}$, Mrutyunjay Suar ${ }^{1}$

${ }^{1}$ School of Biotechnology, KIIT University, 751024, Bhubaneswar, India

${ }^{2}$ Condensed Matter Theory Group, Materials Division, Department of Physics and Astronomy, Uppsala University, Box 516, SE-75120, Uppsala, Sweden

${ }^{3}$ Mads Clausen Institute, NanoSYD, University of Southern Denmark, Alsion 2, DK-6400 Sønderborg, Denmark.

${ }^{4}$ Applied Materials Physics, Department of Materials Science and Engineering, Royal Institute of Technology (KTH), SE-100 44 Stockholm, Sweden

Submitted: January 25, 2021

Accepted: March 22, 2021

Published: March 28, 2021

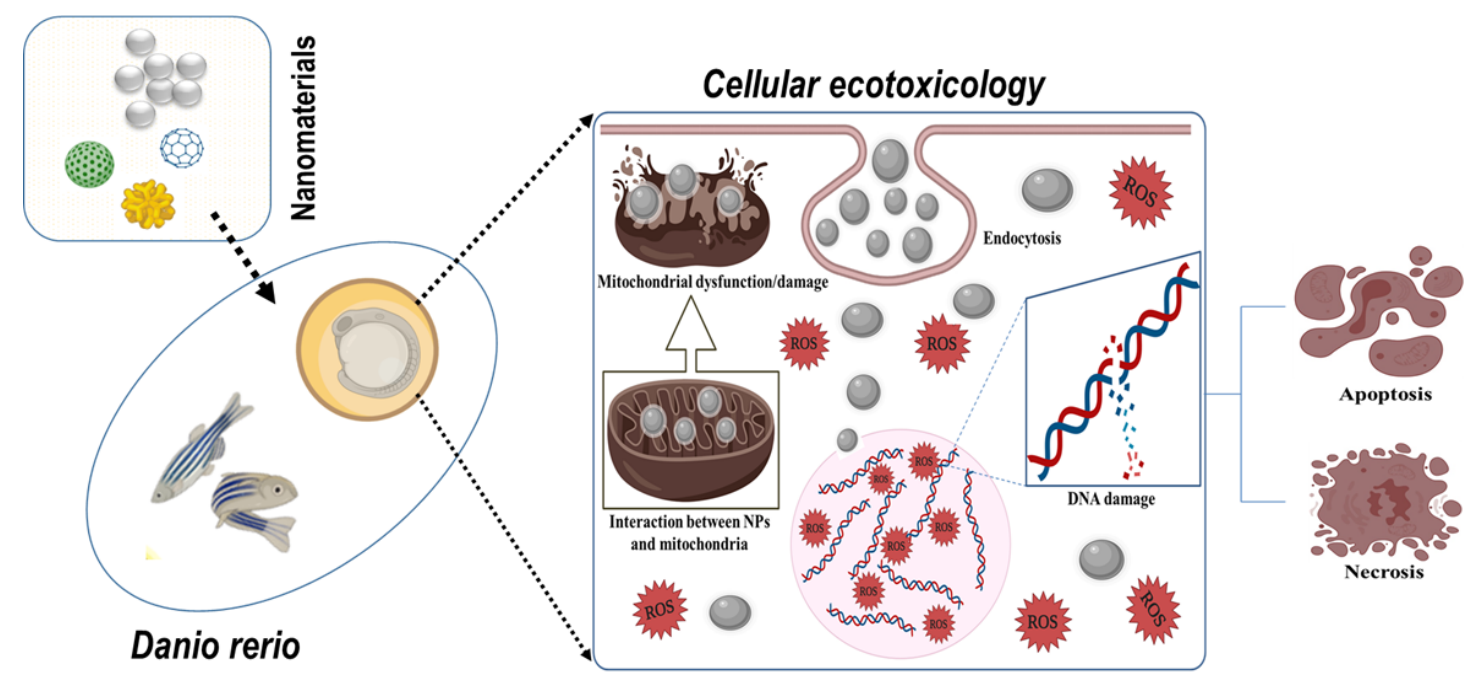

Abstract

With the advancement of technologies, the evolution of nanoscience is occurring at a rapid pace. The demand for nanomaterials in various industries has increased. However, they may pose an increased risk upon exposure to living organisms, demanding toxicological studies. Although risk assessment of nanomaterials is a challenge due to their varieties in sizes and variability in properties, different methodologies of toxicity screenings have been used successfully to evaluate the potential risks. Although nanotechnology has contributed to technology development and well-being, information in these fields is still incomplete. Fortunately, those areas are under continuous research, and there are increasing experimental efforts in determining the environmental effects on humans exposed to nanoparticles. Since the field of nanotechnology is continuously expanding, we need medium- to high throughput nanotoxicity screenings to determine any potential risks caused by nanomaterials. Zebrafish, a well-established model for mammalian research, have advantages when used in these toxicity screenings. This review describes various kinds of toxicities induced in zebrafish embryos by different kinds of nanoparticles. A significant number of experimental data from toxicological studies are also presented that determine nanomaterials' effects, which can be modulated or further mitigated to create a positive impact on living organisms.

Keywords: Zebrafish, Toxicity, Nanomaterial, Engineered nanomaterials

*Corresponding author: sureshverma22@gmail.com, msbiotek@yahoo.com § Authors with equal contributions 


\section{Rationale and Purpose}

The purpose of this review to detail risk assessments of natural and engineered nanomaterials using zebrafish as a model. We highlight the findings and summarize knowledge that has recently been gained of the toxicity of nanomaterials using zebrafish as in vivo model.

\section{Introduction}

The planet has been dealing with a big problem with dangerous chemicals in the atmosphere for many decades. Furthermore, such poisonous substances result in a significant depletion of our natural resources. The only way to get out of such a horrible situation is to conduct rapid screening and characterization of naturally or artificially occurring substances in nature. Cell lines were crucial in the screening of drugs, but cell lines can only screen cell-autonomous phenotypes. The discovery of several model organisms (e.g., Caenorhabditis elegans, Drosophila melanogaster, Xenopus laevis, the laboratory mouse) have contributed to the advancement of testing for toxicological determination. Among all the model organisms, the zebrafish (Danio rerio) model has become an excellent system for studying toxicological sciences. This model system is a tropical teleost fish from the family Cyprinidae, originating from the Ganges River system, Burma, the Malakka peninsula, and Sumatra. Besides that, the zebrafish grows in a short period of around three months at $26^{\circ} \mathrm{C}$ aquatic temperature. (Braunbeck and Lammer, 2006). The zebrafish can adapt to environmental factors like temperature and $\mathrm{pH}$ (Sousa Borges et al., 2020). This model organism also carries some unique features like transparent embryos, rapid development, elevated fecundity rate (200-300 eggs), accessibility of gene manipulation, low expenditure. In toxicity-based assays, zebrafish' transparent feature facilitates the easy monitoring of toxic effects of substances such as teratogenicity, lethality, and reproductive toxicity (Bai and Tang, 2020). The adult zebrafish' size is approximately $3-5 \mathrm{~cm}$; it facilitates their maintenance in a small space with low expenditure. Apart from the advantage of low-cost husbandry, the species' minute size helps perform the experiments with low quantities of dosing solutions (drugs, chemicals, pollutants) that ultimately lower the experiment expense (Hill et al., 2005). The screening of zebrafish genetic map shows 400 different genes and $>2000$ microsatellite markers in zebrafish (Chakraborty et al., 2016). Most interestingly, $70 \%$ of gene expression in the zebrafish model is similar to that of humans (Howe et al., n.d.). Such higher genomic similarity helps in the validation of genotoxicity and developmental toxicity studies using this model system. Along with the genomic similarities, this model system shares similar organ systems with humans (Haque and Ward, 2018a). The functioning of organ systems like the nervous, cardiovascular, and digestive systems is similar to humans (Hsu et al., 2007). Additionally, the cell line assays do not support the pharmacodynamics and pharmacokinetic characterizations of drugs or substances (Jeevanandam et al., 2019). While major pharmacodynamics and pharmacokinetic characterizations of the substances involve metabolism, distribution, excretion, absorption, and toxicity assessment, zebrafish provides accessibility for the determination of all these parameters as an in vivo model. Henceforth, this vertebrate model organism makes way for pharmacological sciences to conduct the testing compounds' preclinical trials. Overall, zebrafish is a vertebrate system that facilitates the advantage of high throughput screening along with a cost-effective experimentation setup.

In the recent scientific period, nanotechnology has contributed to the growth and progress of the worldwide economy. Nanotechnology has generated a cumulative impact on industries associated with electronics, chemistry, and medicine. Such an enormous demand for the field of nanotechnology has turned it out to a multibillion-dollar business sector. Nanotechnology comprises the designing, development, and application of materials at the measurement scale of a nanometer $(\mathrm{nm})$. Therefore, as per ISO (International Organization for Standardization), the size of the nanomaterials (NMs) ranges from $10 \mathrm{~nm}$ to $100 \mathrm{~nm}$ and larger particles up to 1000 to $2000 \mathrm{~nm}$ ("ISO - ISO/TS 19590:2017 - Nanotechnologies — Size distribution and concentration of inorganic nanoparticles in aqueous media via singleparticle inductively coupled plasma mass spectrometry," n.d.). Nowadays, nanomaterials play a key role in the human health and biomedical research fields. Some nanomaterials are utilized for drug delivery to treat lethal diseases 
like cancer. Nanomaterials (NMs)/nanoparticles (NPs) may possess piezo-electric, photoluminescence, optical, and electrochemical properties (Das et al., 2013; Haque and Ward, 2018a). Apart from drug delivery NPs are also used for medical purposes like tissue engineering, bioimaging, and other therapeutic applications like photoablation (Das et al., 2013; Haque and Ward, 2018a; McNamara and Tofail, 2015). The NMs / NPs involved in such wide-ranging applications are further characterized as organic and inorganic NPs ("organicand-inorganic-nanoparticles," n.d.).

Further, organic NPs are classified as dendrimers, layered biopolymers, liposomes, whereas inorganic nanoparticles are classified into materials such as carbon nanotubes, mesoporous silica, gold shell, iron oxide shell, quantum dots (Silva et al., 2019). Scientists have discovered various characteristic features of all the classified NMs and their vital role in the betterment and advancement of nature and society.

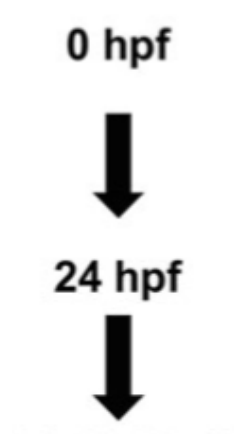

24-120 hpf

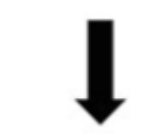

$120 \mathrm{hpf}$

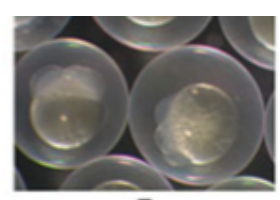

4

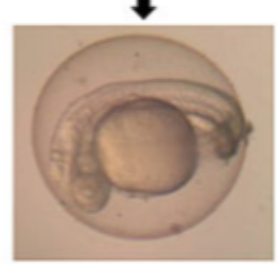

$\Rightarrow \frac{\text { 을 }}{\text { 은 }}$

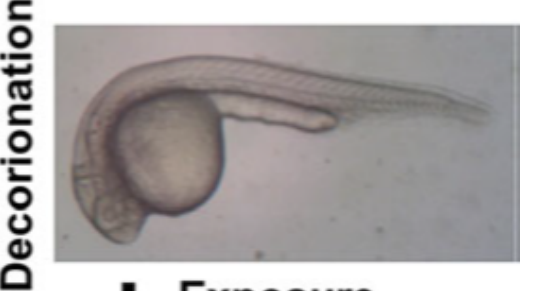

- Exposure

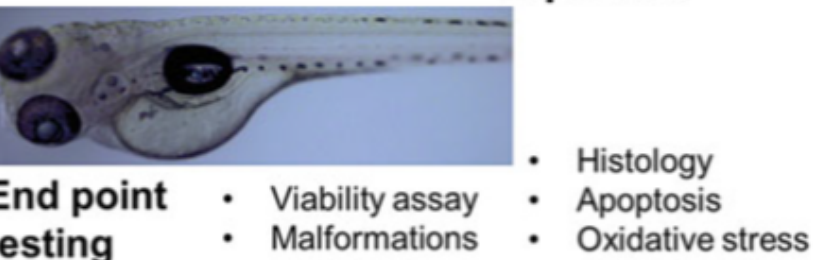

\section{Eggs collected}

Figure 1. Schematic representation of the main procedure steps during zebrafish embryo nanotoxicity assessment, including initial preparation, exposure period, and end point analysis [Figure adapted from (Dumitrescu et al., 2019)].

In toxicology, the median toxic dose $\left(\mathrm{TD}_{50}\right)$ of a drug or toxin is the dose at which toxicity occurs in $50 \%$ of cases, which is useful in half of the population. The type of toxicity should be specific for this value to have meaning for a practical purpose. Zebrafish embryo is emerging as an essential tool for behavioral analysis as well as toxicity testing. In this review, we have shown how toxicity testing $\left(\mathrm{TC}_{50}\right)$ is effec-
However, the environmental emancipation of nanomaterials may lead to potential risks. For instance, the therapeutic application of NMs encourages the non-toxic properties of the materials used, or else the toxicity raised by NMs may further give rise to other health risks. As discussed earlier, we know that NPS assists in drug delivery mechanisms. To escape undesirable circumstances, it is also essential to monitor out the toxic effects of the NPs. Apart from resulting in toxic effects in humans, the environmental exposure of NPs can affect and cause an unwanted loss in nature. It is important to focus on the assessment based on the biocompatibility of NPs and the cytotoxic effects responsible for it. Along with the ecotoxicological concern, the zebrafish model with human homology can be utilized for the evaluation of the starting point of the risk assessments for human health. This review focus on the role of zebrafish as a model for the biocompatibility and toxicological screening of the inorganic nanoparticles (Figure $1)$.

tive in zebrafish from the perspective of nanomaterials. Several biochemical assays and fish embryo toxicity (FET) tests were performed in zebrafish embryos after treatment with different doses of particular nanoparticles. In addition to that, a strong positive correlation between the toxic amounts $\left(\mathrm{TC}_{50}\right)$ calculated in zebrafish embryos and lethal doses $\left(\mathrm{LD}_{50}\right)$ in rodents attained from another database has been observed (Basnet et al., 2017). When taken together, all 
these findings reveal the usefulness of zebrafish embryos as an in-vivo model for behavioral and toxicity testing of nanomaterials and other related compounds. Toxicity profiling of nanoparticles using the zebrafish model system is a broad scientific field. Therefore, the remainder of this review will be focusing on the toxicity assessments of some significant metal and carbon-based nanomaterials on the zebrafish model.

\section{Summary of relevant literature:}

Standardization of zebrafish for nanotoxicology assessments

In terms of toxicity-based assessments, the Organization for Economic Cooperation and Development (OECD) has developed guidelines related to utilizing the zebrafish and other fish species. Several guidelines for Fish Embryo Toxicity (FET) testing (Figure 2),

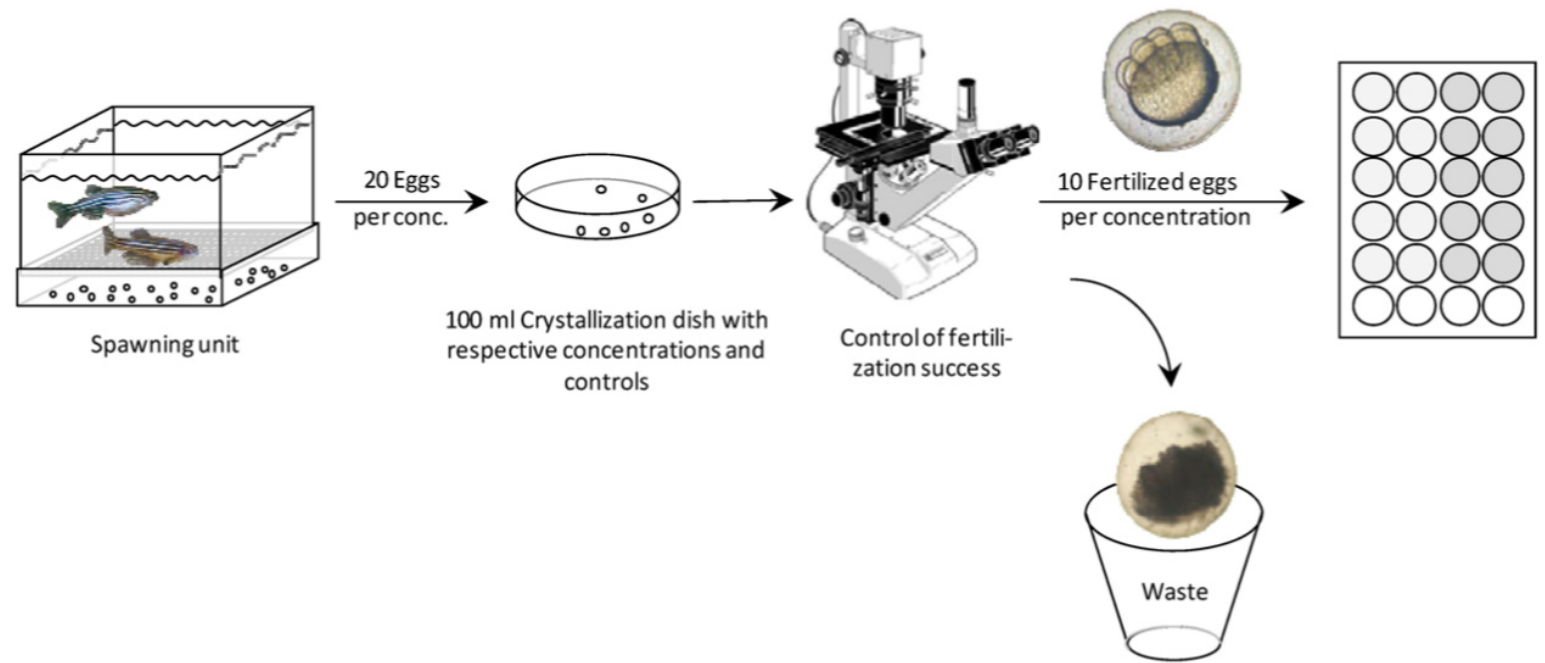

Figure 2. Scheme of the fish embryo toxicity test procedure (from left to right): collection of the eggs, pre-exposure of the eggs immediately after fertilization in crystallization dishes, selection of fertilized eggs with an inverted microscope or binocular and distribution of the fertilized eggs into prepared 24-well microliter plates [Figure adapted from (Lammer et al., 2009)]

Fish: Juvenile Growth Test, and the adultbased Fish: Acute Toxicity Test (Busquet et al., 2014; OECD GUIDELINE FOR TESTING OF CHEMICALS 2 Draft revised version, 2018). The guidelines embrace FET testing for performing the toxicity test about EPA (Environmental Protection Agency) and OECD for the chemical substances, and about the FDA (Food and Drug Administration) and ICH (International Conference on Harmonization) concerning the pharmaceutical products $(O E C D / O C D E$ 2 , n.d.). FET shows a strong and reproducible methodology, and it has been validated by independent laboratory testing (Busquet et al., 2014). The scientific communities and the researchers are advised to follow the standardized guidelines while treating or exposing the zebrafish model for the toxicity testing of harmful chemicals or substances. To decrease the experimental tests' impact on live animals, the European Community provided some suggestions (Brundo and Salvaggio, 2018; Embry et al., 2010; Pecoraro et al., 2018). According to them, an alternative approach for acute toxicity testing is the zebrafish embryo toxicity test (ZFET) since both of them provide the same specificity and sensitivity for the discovery. Whereas ZFET assessment may not be a prominent one for evaluating developmental malformations occurring after the $96 \mathrm{hpf}$ (hours postfertilization). For instance, depending on the normal situation, ZFET does not support the evaluation of skeletal abnormalities, and it is so because the process of calcification in zebrafish begins on the seventh day of development. For the evaluation of toxicity triggered due to methylxanthine drugs, a recent investigation using the FET test was performed in the zebrafish model system. The results of the investigation indicated a robust correlation between the $\mathrm{TC}_{50}$ (threshold concentration) measurements of teratogenicity, morphological defects, and mortality in embryonic zebrafish and the published $\mathrm{LD}_{50}$ (lethal dose) values of mammals. Screening and investigation of a larger amounts of nanoparticles can be performed using the fish embryonic and larval assays. Different types of assays and their results obtained 
from the toxicity evaluation in the zebrafish model have been mention in Table S1 of this review. Following the standardized guidelines, the zebrafish has become a significant model for assessing toxicity caused by the nanoparticles. Also, following the updated guidelines, all the experiments related to nanotoxicity must be performed in the zebrafish.

\section{Advantages of the Zebrafish model over} other biological model organisms

Zebrafish are durable and relatively easy to maintain since they are naturally found in ponds and rice fields. Their learning environment can be replicated in a laboratory setting (Berman et al., 2003). According to one example, a standard aquarium can hold up to 900 adult zebrafish, which corresponds to 75-150 mice on a standard mouse shelf. Overall, zebrafish husbandry is inexpensive, meets space management requirements, and promotes cost-effective science (Heilesen, 2017). Because of the embryos' small size, they are ideal for large-scale drug testing. In 24, 96 well plates, newly discovered drugs may be diluted to various concentrations. The embryos can be led into the wells' plates using ELISA trays. Via the plasma membrane, embryos absorb test samples from the ambient water medium, and toxicity results are provided within a set time frame (Heilesen, 2017; Horzmann and Freeman, 2016). The first-generation antipsychotics, aripiprazole, clozapine, olanzapine, risperidone, and ziprasidone, were tested in Danio rerio embryos. Parameters such as tail and body malformations, edema, and heartbeat rate were considered because the heartbeat rate is the most popular and serves as a foundation for risk assessment assays (Caballero and Candiracci, 2018).

Anatomically and genetically, humans and zebrafish have a lot in common. Despite morphological differences, zebrafish have the same vital organs as humans, except for the lungs, placenta, and mammary gland (Heilesen, 2017). Organs have the same cell types, and their genes have the same identity and functions. Human genes that fit zebrafish account for $71 \%$ of all human genes, and diseased human genes account for $80 \%$ of zebrafish homology. The brain neurochemistry is highly conserved in vertebrates, and the zebrafish has all of the central neuro mediators systems, such as receptors, transporters, and enzymes (Heilesen,
2017; Horzmann and Freeman, 2016). According to an experiment, when cannabinoids were exposed to the larva at various concentrations, the (LC50) was determined. The movement was targeted using automatic video on day 5 of the behavioral analysis (visual-motor response test). Biphasic response to the dark challenge was observed in embryos after acute exposure. Chronic exposure caused deformities in the tail and body, as well as pericardial edema. Cannabinoids had behavioral effects in zebrafish similar to those published in mammalian papers (Akhtar et al., 2013). Their promising productivity and distinct function enhance zebrafish's uniqueness. The embryo's high fluidity rate, rapid embryonal growth, and transparency enable outside observers to observe the cell cycle and development stages. On a single breeding cycle, providing the ideal temperature, $\mathrm{pH}$, conductivity, and food supplement to a single pair will result in 300 to 600 fertilized eggs (Ali et al., 2011; Berman et al., 2003; Carpio and Estrada, 2006; Heilesen, 2017). Since zebrafish are sexually active all year, their reproducibility makes them an excellent research model. Researchers should plan experiments on a continuous mode because phenotypic shreds of evidence like external fertilization and growth can be visualized/ looked at under an ordinary light microscope, which does not happen in mice, and there are fewer ethical problems with zebrafish embryos.

\section{Nanotoxicity assessments using the zebrafish model}

\section{Metal and metal oxide nanoparticle-based} toxicity profiling in the zebrafish model

As shown in Table S1, numerous investigations reported organ toxicities induced in zebrafish due to nanoparticles. Several metals such as gold, silver, copper, aluminum, platinum serve NPs production, which is further utilized in several industrial and scientific fields. Despite such a wide range of applications, studies using the zebrafish model have shown the toxic effects produced by the metal NPs. Several metal nanoparticles and their toxic effects observed in zebrafish are shown in Table S2. Whereas some essential metal oxides such as Copper oxide, Titanium dioxide, Zinc oxide, and Magnesium oxide also serve the same purpose. Studies related to metal oxides also came up with toxic results. Table S3 focuses on the 
toxicity induced in zebrafish when they are treated with numerous sizes and concentrations of metal oxide nanoparticles. Scientific research has been performed to analyze their cytotoxic effects and the betterment of numerous beneficial applications of metal-based NPs.

\section{Silver (Ag) NPs}

Silver nanoparticles (Ag NPs) are expansively used and studied due to their extensive applications as biosensors, therapeutic agents, antimicrobial agents, cosmetics, clothing, drug delivery systems, medical imaging, and many others (Ahamed et al., 2010; Jin and Ye, 2007). The toxicity assessments focusing on experiments based on chronic and acute exposure of Ag NPs in zebrafish are still in the investigation phase. Numerous studies on the zebrafish model explore the mechanism of toxicity that emerged due to Ag NPs. Like other NPs, the toxicity of Ag NPs also depends on several critical factors. Among all the factors, one of the questionable and interesting factors is the size-dependent toxicity raised by Ag NPs. A study showed that via random Brownian motion, the AgNPs in size range of $30-72 \mathrm{~nm}$ diameters got disseminated into chorionic pores of the embryonic zebrafish, and ultimately, it resulted in a potent toxic effect (Lee et al., 2012). Another study also explained the size-dependent toxicity of Ag NPs in adult zebrafish when exposed to increasing concentrations $(8,45,70 \mu \mathrm{g} / \mathrm{l})$ of $\mathrm{Ag}$ NPs with an average diameter of $25 \mathrm{~nm}$ (Brundo and Salvaggio, 2018; Pecoraro et al., 2017). The authors analyzed the biomarkers of oxidative damage, gene expression in the gut, liver, and gill tissues of zebrafish exposed to $\mathrm{Ag}$ NPs. Additionally, the ICP-MS analysis showed the bioaccumulation of Ag NPs. Lesions of secondary lamellae of the gills were reported in the histological analysis with several toxicity degrees, which included subepithelial edema, lamellar fusion, hyperplasia, and telangiectasia in some cases. Although lesion was not observed in the liver but most importantly of the metallothioneins, 1(MTs 1) were highly expressed in zebrafish treated with Ag NPs. This study explains that as compared to the concentrations of Ag NPs, the size and state of aggregation are highly linked with the Ag NP toxicity. Alternatively, Bar-Ilan et al. treated embryonic zebrafish with Ag NPs in size range of $(3,10,50$, and $200 \mathrm{~nm})$, and the observations showed that after $120 \mathrm{hpf}$ (120 hours post-fertilization), there was size-independent 100\% mortality (Bar-Ilan et al., 2009). Another study also showed the size-dependent toxicity property of Ag NPs; in this study, the uptake of 4 $\mathrm{nm}$ Ag NPs was relatively more efficient than $10 \mathrm{~nm} \mathrm{Ag} \mathrm{NPs} \mathrm{(Xin} \mathrm{et} \mathrm{al.,} \mathrm{2015).} \mathrm{The} \mathrm{study}$ showed superior accumulation of Ag NPs in the head of the zebrafish compared to the trunk portion. The charge characteristics and surface properties have also affected the intensity of toxicity. An investigation illustrated that $\sim 12$ $\mathrm{nm} \mathrm{Ag} \mathrm{NPs} \mathrm{functionalized} \mathrm{with} \mathrm{peptides} \mathrm{of} \mathrm{var-}$ ious charges were exposed to positively and negatively charged Ag NPs (Lee et al., 2013). The experiment resulted in deformity and a higher mortality rate in embryos treated with negatively charged peptide-coated Ag NPs. Another study explained the toxicity produced by PVP (polyvinylpyrrolidone) - surface coated Ag NP where the evaluation of larval zebrafish was done based on swimming behavior and responses to different lightning conditions 24 hours post-exposure period (Powers et al., 2011). The experiment reported larger-sized Ag NP - PVP (50 NM) leads to hyperactivity, whereas decreased locomotor activity was led by small Ag NP - PVP $(10 \mathrm{~nm})$. Another investigation by Liu et al. showed toxicities caused due to Ag NPs surface coated with PVP and sodium citrate and found that sodium citrate coated Ag NPs caused higher toxicity than PVP - coated ones (Liu et al., 2019). The evaluation of oxidative stress plays an important role in determining toxicity given rise by any nanomaterials or nanoparticles. Several studies and experiments based on the oxidative stress led by NPs have been performed in the zebrafish model system. For instance, the assessment of apoptosis and oxidative stress in the zebrafish liver led to the conclusion that Ag NPs can also lead to hepatotoxic behavior (Choi et al., 2010). The experiment reported that the up-regulation of $p 53$-related pro-apoptotic genes such as $p 21$, $B a x$, and Noxa led to apoptotic changes along with disrupted hepatic cell cords in the zebrafish exposed to Ag NPs. On the contrary, another experiment showed fragile endoplasmic reticulum stress in embryonic zebrafish led by Ag NPs along with all endoplasmic stress responses in vitro in the liver cells of zebrafish (Choi et al., 2010). The DNA repair process and the cell cycle arrest were led by the activation 
of p53 target genes, whereas apoptosis was stimulated due to prolonged activation of $p 53$. Various methods have been developed for the reduction of the toxicity produced by Ag NP. Studies had shown that reduced oxidative stress had been obtained when the embryonic or adult zebrafish were treated with sulfidation and cysteine-coated Ag NP (Devi et al., 2015; George et al., 2012). An investigation by Verma et al. (Figure 3 (a)) demonstrated the biosynthesis of Ag NPs via culture supernatant of two grampositive (B. thuringiensis and $S$. aureus) and two gram-negative (E. coli and Salmonella typhimurium) bacterial strains (Verma et al., 2018a).

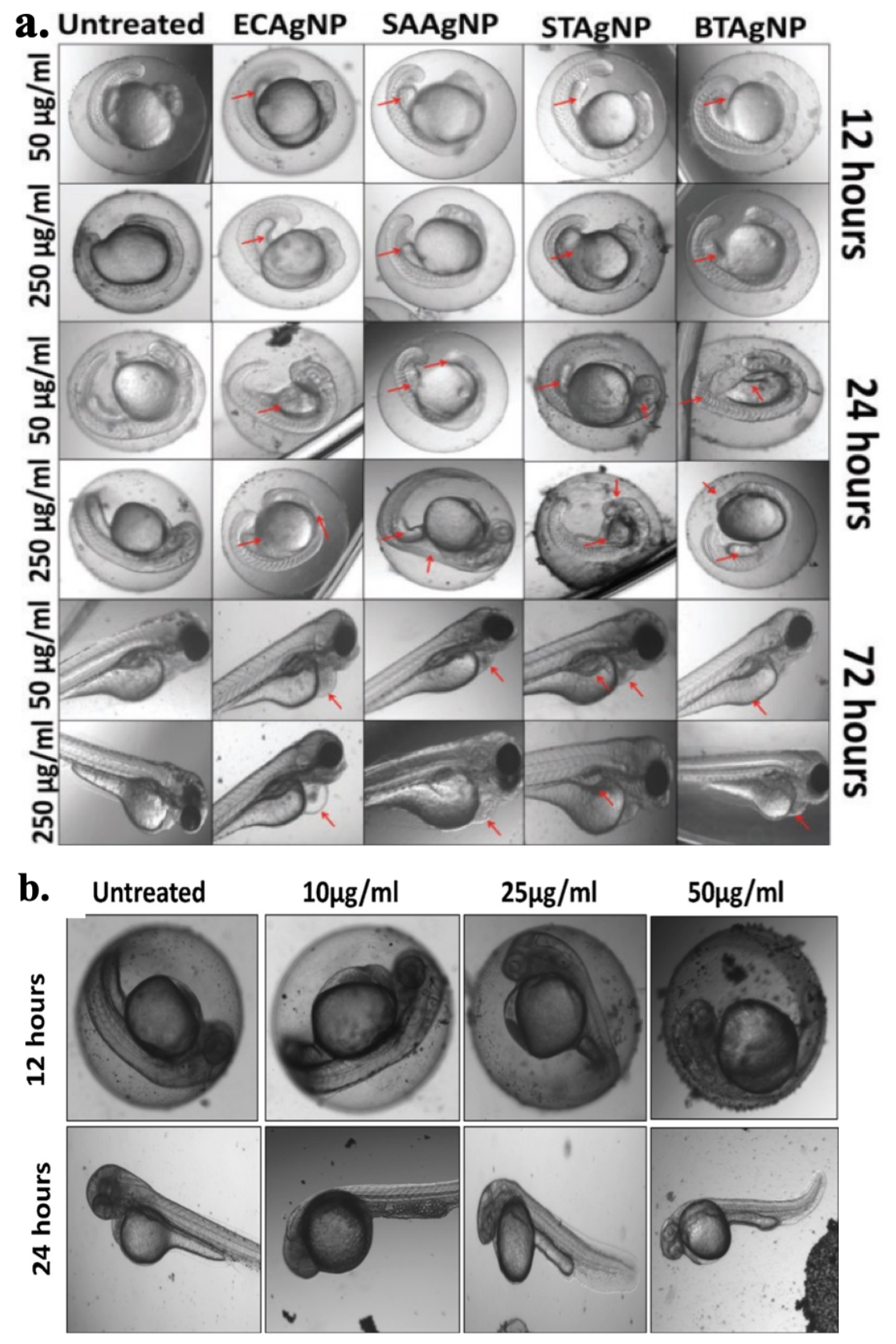

Figure 3. (a) Morphological and cellular changes in zebrafish embryo treated with different biosynthesized silver nanoparticles. Embryos were treated with 50 and $250 \mathrm{mg} / \mathrm{ml}$ concentration of nanoparticles for 12, 24, and $72 \mathrm{~h}$. Images were taken with bright field microscope. Arrow indicates morphological abnormalities like pericardial edema and swelled gastrointestinal lumen in head and trunk region, respectively [Figure adapted from (Verma et al., 2018a)]. (b) Bright field images of morphological analysis of zebrafish embryos exposed to different concentration of green synthesized AuNP [Figure adapted from (Kumari et al., 2019)] 
In addition, the results demonstrated that $\mathrm{Ag}$ NPs led to abnormalities like pericardial edema and swelled gastrointestinal lumen in the head and trunk region. Several studies and experiments explain the unwanted consequences led by Ag NPs, and therefore further research may clear out the understanding of several parameters or factors affecting the toxicity rate of $\mathrm{Ag}$ NPs.

\section{Gold (Au) NPs}

Due to the unique optical properties and chemical stability, the nanoscale application of $\mathrm{Au}$ NPs has gained higher demand in biomedical sciences (Chakraborty et al., 2016). Au NPs are widely utilized in cellular labeling and imaging, drug delivery, and diagnostic of diseases such as cancer, diabetes, and Alzheimer's (Chakraborty et al., 2016; Li and Chen, 2011). In a brief review, Yeh et al. showed the essential features of Au NPs, such as the large surface-to-volume ratio, size, and shape-related optoelectronic properties, lower toxicity, and excellent biocompatibility (Yeh et al., 2012). The same authors stated that the Au NPs possess the properties like redox activity which is used in electrochemical sensing and electronic devices, fluorescence quenching used in sensor fabrication and material science, surface plasmon resonance (SPR) in Colorimetric sensing and photothermal therapy, surface-enhanced Raman scattering used for imaging and sensing. Despite such extensive useful properties and applications, the Au NPs may be responsible for causing potential damage to living organisms. However, in the same place, studies have shown that Au NPs may also be responsible for triggering cytotoxicity in humans (Gerber et al., 2013; Goodman et al., 2004). The toxicity of $\mathrm{Au}$ NPs shown in zebrafish depends on several factors such as surface coating, particle shape, and surface charge (Bai and Tang, 2020). Severe problems like genome alterations and cellular dysfunctions were observed when adult zebrafish were treated with food comprising $\mathrm{Au}$ NPs (12 and $50 \mathrm{~nm}$ ) (Geffroy et al., 2012). A study demonstrated the toxicity and biodistribution study of modified Au NPs (Sangabathuni et al., 2017). The adult zebrafish were treated with three diverse-shaped Au NPs (rod, sphere, and star), and the observation stated that there was amplified uptake and clearance rod-shaped $\mathrm{Au}$ NPs. Another study showed that accumula- tion of $14 \mathrm{~nm}$ Au NPs within adult zebrafish tissues leads to augmented oxidative stress resulting in unwanted genome modification in assorted tissues. Also, Au NPs possess higher toxicity compared to when compared with ionic $\mathrm{Au}$ (Dedeh et al., 2015). Another critical determinant for the toxicity profiling of Au NP is its surface charge functionalization. An experiment showed the study of lethality and developmental toxicity caused by positively and negatively charged Au NPs in embryonic zebrafish (Tanguay et al., 2011). Moreover, the result showed that positively charged Au NPs led to mortality, whereas negatively charged Au NPs caused malformations in zebrafish embryos. Another author also showed similar results when zebrafish embryos were treated with $\mathrm{Au}$ NPs coated with three different charges, i.e., positive, negative, and neutral (Truong et al., 2012). Also, the experiment showed toxic effects like hypo - locomotor activity and high lethality, while neutrally coated Au NPs showed no effects. An assessment demonstrated minor malformations but significant mortality in zebrafish when they were treated with positively charged $\mathrm{N}, \mathrm{N}, \mathrm{N}$ - triethylammoniumethanol (TMAT) (Haque and Ward, 2018a). The same study also showed that the treatment of zebrafish with negatively charged 2-mercaptoethanatesulfonate (MES) did not cause significant mortality within the exposure period of five days. But no adverse effects were observed when zebrafish were treated with the Au NPs functionalized with a higher concentration (up to $250 \mathrm{ppm}$ ) of Neutral 2,2 mercaptoethoxyethoxyethanol (MEEE) and 2,2-mercaptoethoxyethanol (MEE). Kim et al. further investigated the developmental toxicity resulted from positively charged Au NPs (1.3 nm), which were functionalized with TMAT (Kim et al., 2013). The experiment showed toxicity effects like abnormalities in the development of eyes and behavioral changes and altered pigmentations. Browning et al. showed dose-dependent toxic effects of $\mathrm{Au}$ NPs in embryonic zebrafish (Browning et al., 2009). The results stated that with an increase in the concentration of $\mathrm{Au}$ NPs, the accumulation of Au NPs was also increasing. The Au NPs had accumulated in the embryos via chorionic space, but the effects of $\mathrm{Au}$ NPs were not proportionally associated with the concentration. Apart from various properties like surface charge, surface coating, and 
particle shape or size, the biocompatibility of $\mathrm{Au}$ NPs also depends on its synthesis process. Interestingly, the green synthesis of nanoparticles has gained the scientific community's attention and has thrown a major impact on the field of nanotechnology. Extraction of some metal and metal oxide nanoparticles from various plant extracts have been described in Table S4. A recent study by S. Kumari et al. reveals the successful synthesis and characterization of $\mathrm{Au}$ NPs with the help of Andrographis peniculata and its cytotoxic effects on embryonic zebrafish (Kumari et al., 2019). The study demonstrated the morphological and developmental changes caused by $\mathrm{Au}$ NPs at the $\mathrm{LC}_{50}$ value of $116 \mu \mathrm{g} / \mathrm{ml}$ (Figure 3 (b)). Internalization and accumulation of green synthesized $\mathrm{Au}$ NPs in the chorion of embryos led to morphological changes such as malfunctioned eyes, edema, and bending of the tail. Additionally, observations such as alteration in hatching rate and the mortality rate were also made. Overall, studies related to $\mathrm{Au}$ NPs using the zebrafish model system explain the various parameters on which the biocompatibility and toxicity depend upon. Henceforth, further studies are required to explore the benefits and disadvantages of Au NPs.

\section{Copper ( $\mathrm{Cu}$ ) and copper oxide (CuO)}

The copper $(\mathrm{Cu})$ NPs are indulged in several applications, including petroleum lubricants, catalysts, some consumer products of the pharmaceutical industry, gel propellants, sintering active agents, and also as active combustion agents (Adeleye et al., 2016; Dankovich and Smith, 2014; Lee et al., 2016). Metallic copper nanoparticles possess unique properties such as high stability against aggregation, hydrophilic character, and biocompatibility (Tamilvanan et al., 2014). Besides the wide range of applications and unique features, numerous studies using the zebrafish model have disclosed the toxicity produced by $\mathrm{Cu}$ NPs (Figure 4) (Bai and Tang, 2020).

In a study by Kovrižnych et al., it was found that the mortality rate in zebrafish increases with an $\mathrm{LC}_{50}$ value of $3 \mathrm{mg} / \mathrm{L}$ when they are exposed to $\mathrm{Cu}$ NP (Kovrižnych et al., 2013). Also, another investigation reported that the treatment of zebrafish with $\mathrm{Cu}$ NPs (48- hour $\mathrm{LC}_{50}$ value of $1.5 \mathrm{mg} / \mathrm{L}$ ) leads to acute toxicity since abnormal gill morphologies along with different gene expression patterns were observed (Griffitt et al., 2007). Most importantly, the toxicity of $\mathrm{Cu}$ NPs also becomes questionable since it is hardly conventional that the toxicity is produced by the $\mathrm{Cu}^{2+}$ ions released by $\mathrm{Cu}$ NPs or due to the molecular roles of $\mathrm{Cu} \mathrm{NP}$. An assessment of acute toxicity of spherical Cu NPs $(50 \mathrm{~nm})$ was performed in zebrafish, and it was discovered that the $\mathrm{Cu}^{2+}$ ions are the main factor giving rise to toxicity (Song et al., 2015). Also, two different research groups performed a comparative study of $\mathrm{Cu}$ NPs with soluble $\mathrm{Cu}^{2+}$ ions in zebrafish (Griffitt et al., 2007; Hua et al., 2014a). Both studies revealed that instead of $\mathrm{Cu}^{2+}$ ions, the particle form of $\mathrm{Cu}(\mathrm{Cu} N P s)$ was responsible for the toxicity. The dose or the concentration-dependent factor also plays a key role in the toxicity of $\mathrm{Cu}$ NPs. Studies of toxicity caused by $\mathrm{Cu}$ NPs in zebrafish came up with the result that higher concentrations of $\mathrm{Cu}$ NPs led to death at the gastrula stage. In addition, it was also found that $\mathrm{Cu}^{2+}$ ions and $\mathrm{Cu}$ NPs show similar effects on embryonic zebrafish (Bai et al., 2010).

A similar result was obtained when another research group found concentration-dependent - ROS generation the embryonic zebrafish were exposed to $\mathrm{Cu}$ NPs (Denluck et al., 2018). An investigation showed that $\mathrm{Cu}$ NPs inhibited the specification and configuration of three layers of the swim bladder. It was also observed that the Wnt signaling in embryos was down-regulated; interestingly, the inhibiting effects of $\mathrm{Cu}$ NPs on zebrafish swim bladder development were found to be neutralized by Wnt agonist 6 bromoindirubin - 3'- oxime (J. P. Xu et al., 2017). Additionally, a significant reduction in the rheotaxis behavior and moderate reduction of lateral line neuromasts were observed in zebrafish when they were exposed to $\mathrm{Cu}$ NPs (McNeil et al., 2014). Thus, several studies using the zebrafish model validates the toxic effects produced by $\mathrm{Cu}$ NPs, and thereafterproper utilization of $\mathrm{Cu}$ NPs may aid in evading its considerable damage.

The oxide form of $\mathrm{Cu}$ NPs that is the copper oxide $(\mathrm{CuO}) \mathrm{NPs}$ is widely utilized for applications like photocatalysts, antimicrobial agents, gas sensors, high - temperature superconductors, energy transfer fluids, batteries, and agricultural biocides (Batley et al., 2011; Hou et al., 2017; Kim et al., 2012; Llorens et al., 2012). 


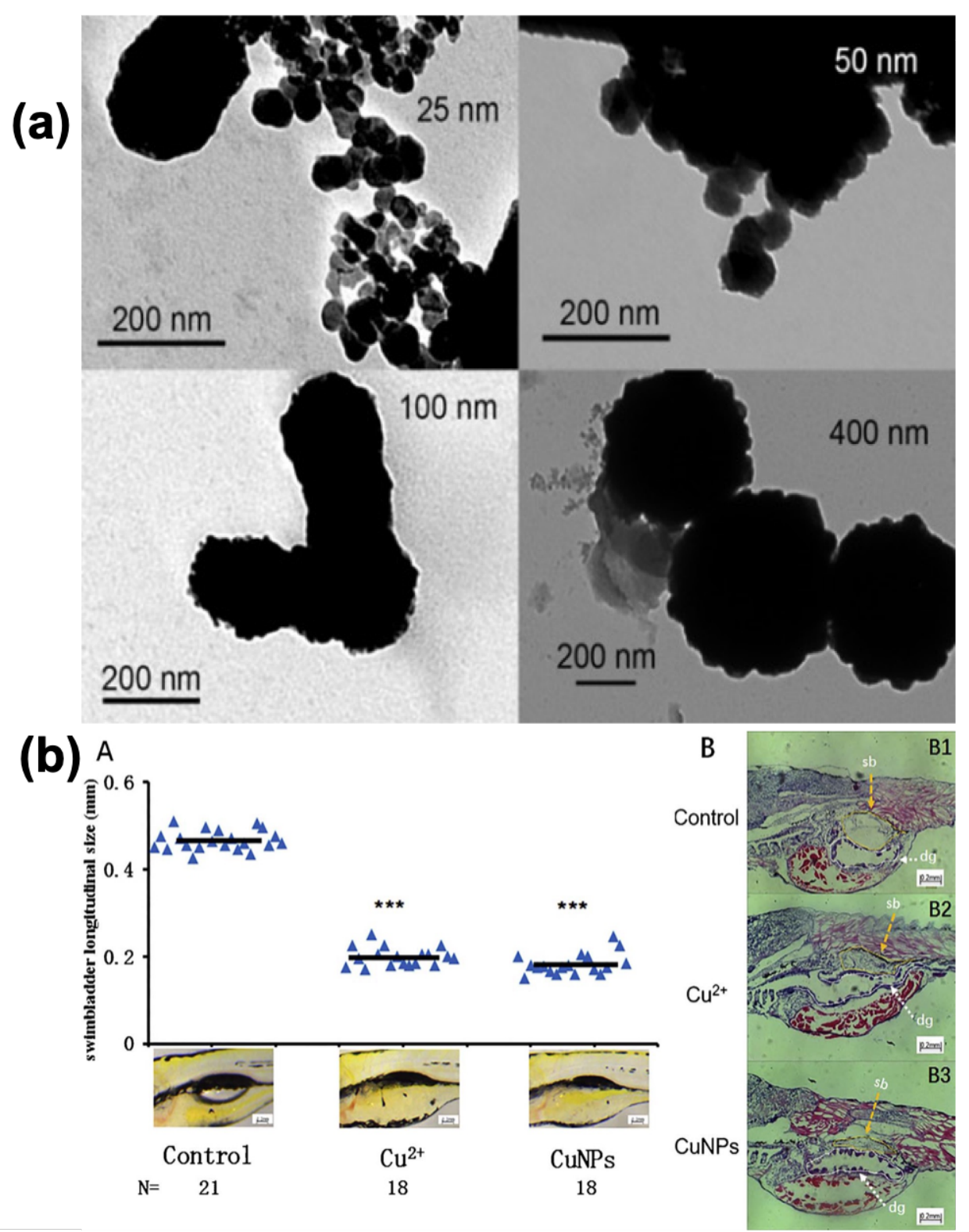

Figure 4. (a) Transmission electron microscopy micrographs of the aggregate of $25 \mathrm{~nm}, 50 \mathrm{~nm}, 100 \mathrm{~nm}$ copper nanoparticles (CuNPs), and $400 \mathrm{~nm}$ sub-micron sized particle (SMPs) [Figure adapted from (Hua et al., 2014a). (b) Cu2+ and CuNPs induced non-inflated swimbladder in zebrafish embryos [Figure adapted from (J. P. Xu et al., 2017)]. (A) Size of swimbladder was reduced significantly in Cu2+ and CuNPs treated embryos. (B) H\&E staining of sagittal sections of embryos from different treatment groups at the swimbladder location. sb, swimbladder, orange dotted lines cycled; dg, digestive gut, white dotted lines cycled. $* * *, * *$ and $*$ indicate $P<0.001,0.01$, and 0.05 , respectively.

The vast utilization of $\mathrm{CuO}$ NPs may cause potential risks to the environment and human health. Numerous studies came up with noteworthy observations of toxicity in zebrafish caused by $\mathrm{CuO}$ NPs. Abnormal phenotypic behavior such as delayed epiboly and smaller eyes and heads were observed in zebrafish when exposed to CuO NPs (J. Xu et al., 2017). An investigation has explained that the zebrafish neuronal differentiation and liver maturation are affected by $\mathrm{CuO}$ NPs (Sun et al., 2016). The experiment results reported that unwanted circumstances like neurotoxicity and hepatotoxicity were observed in embryonic and larval zebrafish when exposed to high doses of $\mathrm{CuO}$ NPs for a shorter period. Hepatic hypoplasia was the indicator for hepatotoxicity, whereas delayed retinal differentiation coupled with abridged locomotor ability indicated the neurotoxicity. Another investigation demonstrated the effects of $\mathrm{CuO}$ NPs on the vasculogenesis of transgenic zebrafish $\mathrm{Tg}$ (nacre/flil: EGFP) (Chang et al., 2015). The results reported that 
the expression of vascular endothelial growth factor was reduced, and apoptosis was initiated, which resulted in inhibition of vasculogenesis caused by $\mathrm{CuO}$ NPs. Studies have also proved that $\mathrm{CuO}$ NPs are responsible for acute exposure effects in developing zebrafish embryos, leading to oxidative stress, subsequently apoptosis, and developmental abnormalities (Ganesan et al., 2016). Kumari and Panda et al. performed the toxicity assessment of green synthesized $\mathrm{CuO}$ NPs in zebrafish embryos (Kumari et al., 2017). The CuO NPs were synthesized from the floral extract of Calotropis gigentea. The treatment of zebrafish with $\mathrm{CuO}$ NPs ranging from lower to higher concentrations resulted in several phenomenons like abnormal yolk sac, pericardial edema, and higher concentration bending of the notochord. The experimental report indicated that the green synthesized $\mathrm{CuO}$ NPs show more biocompatibility than commercially synthesized $\mathrm{CuO}$ NPs. Therefore, further research and studies may lead to discovering novel techniques for the synthesis of $\mathrm{CuO}$ NPs with reduced or no toxic effects on the living system.

\section{Titanium dioxide $\left(\mathrm{TiO}_{2}\right)$}

The admirable optical, magnetic, and electrical properties are the reason behind the sudden increase in titanium dioxide production $\left(\mathrm{TiO}_{2}\right)$ (Subhapriya and Gomathipriya, 2018). Besides, $\mathrm{TiO}_{2}$ NPs are valuable transition metal oxide material that has attracted the researchers for their unique qualities including non-toxic, fine resistance to chemical erosion, simple control, and most importantly low cost (Srinivasan et al., 2019). In our daily life, the $\mathrm{TiO}_{2} \mathrm{NPs}$ have various applications, such as components of shampoos, toothpaste, soap, and pigments in sunscreen (Noman et al., 2019). Studies have demonstrated that when exposing zebrafish to a low dose $(1 \mathrm{mg} / \mathrm{L})$ of $\mathrm{TiO}_{2} \mathrm{NPs}$, then no major developmental malformation is observed (Y. J. Wang et al., 2014). Whereas harmful effects like embryonic malformation and death were observed when zebrafish were treated with high doses of $\mathrm{TiO}_{2} \mathrm{NPs}$ (Chakraborty et al., 2009). Another study also showed that the treatment of zebrafish with higher concentrations of $\mathrm{TiO}_{2}$ NPs led to the accumulation of NPs in the heart, gills, brain, and liver of the zebrafish (J. Chen et al., 2011). Depending on the respective exposure period and concentration range, both the bulk $\mathrm{TiO}_{2}$ and $\mathrm{TiO}_{2}$ NPs have shown toxic effects in zebrafish. For instance, embryonic zebrafish showed morphological and anatomical changes when treated with lower concentrations $(50 \mu \mathrm{g} \mathrm{mL}-1)$ and higher concentrations $(250 \mu \mathrm{g} \mathrm{mL}-1)$ of bulk $\mathrm{TiO}_{2}$ and $\mathrm{TiO}_{2} \mathrm{NPs}$ (Verma et al., 2018b). Furthermore, the experiment resulted in numerous abnormalities such as deformation chorion and yolk sac in $48 \mathrm{hpf}$ (48 hours post-fertilization), deformed tail, notochord, and heart development $96 \mathrm{hpf}$. Several studies have also revealed some vital toxicity effects of $\mathrm{TiO}_{2} \mathrm{NPs}$ in zebrafish, including neurotoxicity, behavioral toxicity, reproductive toxicity, and genotoxicity. Looking into the neurotoxicity behavior becomes important since the $\mathrm{TiO}_{2} \mathrm{NPs}$ are found to play a major role in it. The impact on neurogenesis and neuronal differentiation was observed when embryonic zebrafish were treated with a lower concentration of $\mathrm{TiO}_{2} \mathrm{NPs}$ (Y. J. Wang et al., 2014). On the other hand, significant effects on swimming parameters, average and maximum velocity were observed when larval zebrafish were exposed to $\mathrm{TiO}_{2} \mathrm{NPs}$ (T. H. Chen et al., 2011). Studies have shown that $\mathrm{TiO}_{2} \mathrm{NPs}$ can target the brain of the fish via gaining entry through the blood-brain-barrier which may further lead to the inhibition of $\mathrm{Na}+, \mathrm{K}+-$ ATPase activity (Chakraborty et al., 2009; Ramsden et al., 2009). Investigations came up with results showing the relation between $\mathrm{TiO}_{2} \mathrm{NPs}$ and neurodegenerative diseases like Parkinson's disease. To study the neurotoxicity caused by $\mathrm{TiO}_{2}$ NPs, several researchers selected PC12 cell lines and embryonic zebrafish. Moreover, the results reported that the accumulation of $\mathrm{TiO}_{2}$ NPs in the brain resulted in the generation of ROS and cell death of the hypothalamus. Most importantly, $\mathrm{TiO}_{2} \mathrm{NPs}$ may lead to the formation of Lewy bodies in the brain since the exposure of $\mathrm{TiO}_{2} \mathrm{NPs}$ can activate some Lewy bodies related gene expression, which involves activation of pink1, parkin, alpha-syn, and uchll. The exposure of $\mathrm{TiO}_{2} \mathrm{NPs}$ may be a fundamental risk factor for the development of Parkinson's disease, and it is indicated by the in vivo and in vitro loss of dopaminergic neurons (Hu et al., 2017). Moreover, the results of a comparative study between the bulk $\mathrm{TiO}_{2}$ and $\mathrm{TiO}_{2}$ NPs on the zebrafish brain discovered that $\mathrm{TiO}_{2}$ NPs were more toxic than bulk $\mathrm{TiO}_{2}$ due to enhanced lipid oxidation and degradation 
(Palaniappan and Pramod, 2011). Assessment of reproductive toxicity explained that the $\mathrm{TiO}_{2}$ NPs may interfere with the synthesis of vitellogenin and /or might directly act on the primary follicles (Wang et al., 2011). In addition, after the 13 weeks of $\mathrm{TiO}_{2}$ NPs exposure, it was discovered that the cumulative number of zebrafish eggs was reduced. Another study also showed a similar effect when the lower rate of embryo production was observed after the zebrafish were exposed to $1.0 \mathrm{mg} / \mathrm{L}$ of $\mathrm{TiO}_{2}$ NPs and bulk $\mathrm{TiO}_{2}$ (Ramsden et al., 2013). Investigations have also revealed the effects of $\mathrm{TiO}_{2}$ NPs on zebrafish testis. The experiment reported that autophagy and necrosis at higher doses were provoked by TiO2NPs; additionally the study also showed that the spermatogenic cells and testicular morphology of zebrafish were also affected by TiO2NPs (Kotil et al., 2017). The genotoxicity was also observed in a study when zebrafish were treated with $\mathrm{TiO}_{2}$ NPs for 14 and 21 days, and the DNA damage was observed in the experiment (Rocco et al., 2015). Another experiment showed the effect of TiO2NPs in the gene expression pattern of embryonic zebrafish. The experimental results showed the genes involved in the kinase activity, immune response, circadian rhythm, and vesicular transport were down-regulated (Jovanović et al., 2011). So, the toxicity generation by $\mathrm{TiO}_{2} \mathrm{NPs}$ should be measured and kept into concern, and thereafter it should be executed in the respective fields.

\section{Zinc Oxide ( $\mathrm{ZnO})$}

The zinc oxide $(\mathrm{ZnO})$ NPs are frequently utilized in electrical appliances, ceramics, sunscreens, cosmetics, and photonics, and it is so because of its unique properties such as high isoelectric point, photocatalytic efficiency, antimicrobial property, biocompatibility, and transparency (Mirzaei and Darroudi, 2017). Most importantly, the $1 \mathrm{D} \mathrm{ZnO}$ nanostructures hit upon the remarkable potentials for nanoelectronics and sensing applications; the feature of high surface to volume $(\mathrm{S} / \mathrm{V})$ shown by $1 \mathrm{D} \mathrm{ZnO}$ nanostructures plays a significant role behind it (Mishra and Adelung, 2018). Also, the same authors have reported that the nanostructures of $\mathrm{ZnO}$. The $\mathrm{ZnO}$ nanostructures show some inim- itable piezoelectric properties due to their noncentrosymmetric crystal structure with $\mathrm{Zn}^{2+}$ and $\mathrm{O}^{2-}$ polar surfaces. Thereby $\mathrm{ZnO}$ nanostructures attract smart technologies such as piezophotocatalysis, piezophototronics, and piezotronics. Despite such high throughput applications, the $\mathrm{ZnO}$ NPs are also found to show toxic properties when exposed to zebrafish. For instance, the higher concentrations $(>0.1 \mathrm{mg} / \mathrm{L})$ of $\mathrm{ZnO}$ NPs $(20 \mathrm{~nm})$ led to the delayed development and also inhibited the hatching of embryonic zebrafish (Kteeba et al., 2017; Zhu et al., 2008). Comparative studies related to the toxicity instigated by bulk and nanosized ZnO NPs were performed in various experiments. Moreover, the experiments concluded that equivalent toxic levels were shown when embryonic and larval zebrafish were treated with bulk and nano-sized ZnO NPs (Xiong et al., 2011; Zhu et al., 2008). The same studies revealed that $\mathrm{ZnO}$ NPs were found to exert dose-dependent toxicity. The $\mathrm{ZnO}$ NPs are known for the generation of ROS and oxidative stress. Studies have shown that zebrafish' gill and liver were the main target organ that was damaged by the effects of oxidative stress triggered by ZnO NPs (Xiong et al., 2011). The same research also demonstrated the elevation of malondialdehyde content in the gills and livers of zebrafish. Verma et al. performed an investigation on in vivo cytotoxicitybased assessment of industrially synthesized $\mathrm{ZnO}$ NPs, and the embryonic zebrafish were exposed to bulk $\mathrm{ZnO}$ and $\mathrm{ZnO}$ NPs (Verma et al., 2017). The results reported that the development of embryos was affected by bulk $\mathrm{ZnO}$ and $\mathrm{ZnO}$ NPs since ROS generation (Figure 5) in conjunction with deformities in the eye, tail, and yolk was visualized in both cases. Another assessment showed toxicity produced in embryonic zebrafish treated with differently shaped ZnO NPs, such as nanosticks, nanospheres, and cuboidal submicron particles (Hua et al., 2014b). Concerning the hatching retardation and mortality endpoints, it was found that stickshaped $\mathrm{ZnO}$ NPs were more toxic. Along with developmental toxicity and oxidative stress, $\mathrm{ZnO}$ NPs are also known to cause DNA damage (Zhao et al., 2013). ZnO NPs lead to the downregulation of genes such as Bcl-2, Nqo1, and Gstp2, related to oxidative damage, ROS production, and DNA damage. 


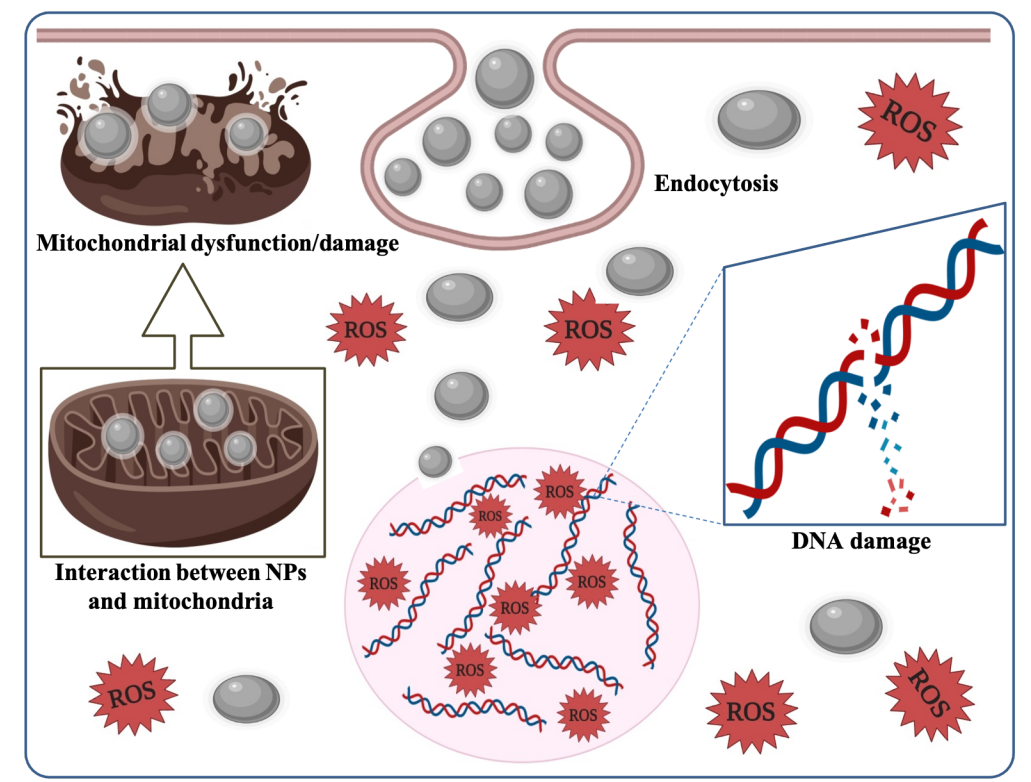

Figure 5. Mechanism of cytotoxicity of metal nanoparticles (NPS). ROS reactive oxygen species regulation, apoptosis, and necrosis. In view of these reports, the in vivo toxicity can be described as an effect of dysregulated expression of ROS production, leading to greater apoptosis and other cell death-related phenomena (Patel et al., 2019; Verma et al., 2019).

Again, the surface coating properties also appear to be an important parameter for studying the toxicity caused due to ZnO NPs. For instance, Girigoswami et al. studied the toxic effects of $\mathrm{ZnO} \mathrm{NPs}$, ZnO NPs coated with chitosan ( $\mathrm{ZnO}$ - CTS), and ZnO NPs coated with polyethylene glycol ( $\mathrm{ZnO}$ - PEG) on the zebrafish embryos (Girigoswami et al., 2015). The results reported that reduced deposition of $\mathrm{ZnO}$ - CTS, and ZnO - PEG NPs were found atop the zebrafish eggs. Moreover, the study also showed a higher survival rate of zebrafish embryos treated with $\mathrm{ZnO}$ - CTS. Another study showed higher biocompatibility of polymer-coated $\mathrm{ZnO}$ NPs as compared to spherical $\mathrm{ZnO}$, and the hatching was immensely affected by the leaf-shaped ZnO NPs (Ong et al., 2014). Whereas investigations have reported that the release of $\mathrm{Zn}^{2+}$ ions was primarily responsible for the toxicity of $\mathrm{ZnO}$ NPs (Boran and Ulutas, 2016; Brun et al., 2014). Henceforth, additional studies are required to bring out the detailed mechanism of toxicity given rise by the $\mathrm{ZnO}$ NPs.

\section{Toxicity profiling of Carbon-based nanoparticle using the zebrafish model}

Followed by numerous metal-based nanomaterials, carbon-based nanomaterials are frequently utilized in consumer products (Brand et al., 2020). Due to the several applications in therapeutics, cell and tissue labeling, drug de- livery system, and tissue scaffold reinforcements, carbon-based nanomaterials have gained a mounting interest in the field of biomedical research (Cha et al., 2013). As compared to other NPs, the carbon-based NPs are more promising since it has shown lower toxicity (Rani et al., 2018). Over the past few years, the zebrafish model system has been utilized for several toxicity assessments related to carbonbased nanoparticles such as carbon nanotubes and fullerenes.

\section{Carbon nanotubes (CNTs)}

In 1991, Sumio Iijima synthesized the nanosized hollow cylindrical form of carbon, popularly known as carbon nanotubes (CNTs) (Iijima, 1991; Meyyappan, 2005). In the present scenario of nanotechnology, the CNTs belong to the most commercially relevant classes of nanomaterials. The CNTs belong to the most commercially relevant nanomaterials classes due to the broadest range of applications, from composites to energy storage, health care, consumer electronics, and many others (Ema et al., 2016). A seamless cylinder is formed using the rolled sheet(s) of graphite, which further forms CNTs. Further classifications of CNTs are done into two major classes known as single-walled CNTs (SWCNTs) and multi-walled CNTs (MWCNTs). As the name suggests in both types, a single sheet of graphite is utilized to form the cylinder of SWCNTs, whereas, in MWCNTs, numerous sheets are rolled for the 
formation of a concentric tube (MonteiroRiviere et al., 2007). The CNTs possess unique chemical and physical characteristics, due to which they have gained the attraction of several biomedical applications (Cha et al., 2013). Such unique characteristics are accountable for their utilization in chemotherapeutic agents' delivery, including doxorubicin and paclitaxel, small interfering RNAs, antibodies, and genes (He et al., 2013; Liu et al., 2009). In contrast, the investigations have demonstrated the toxic effects produced by various forms of CNTs. To avoid such toxic effects, the toxicity evaluation of CNTs is also an obligatory step that must be performed before their extensive commercial utilization. For instance, in a study, CNTs were found to Also, four hpf (blastula stage) embryonic zebrafish were exposed to SWCNTs
(Cheng et al., 2007). It was found that the microscale SWCNTs agglomerates have adhered to the outer layer of the chorion. The SWCNTs were responsible for the delayed hatching of embryos, whereas other factors involving cellular development, hatching success, survival rate, or morphological, molecular development of embryos were not affected by SWCNTs. Another research group reported that CNTs were responsible for alterations in the zebrafish brain and gonads ( $\mathrm{Li}$ et al., 2015). Liu et al. performed a comparative toxicity-based study of Multi-Walled Carbon Nanotubes (MWNTs), Graphene Oxide (GO), and Reduced Graphene Oxide (RGO) on zebrafish embryos (Figure 6) (Liu et al., 2014).



Figure 6. Effect of exposure to different concentrations (control, 1, 5, 10, 50, and $100 \mathrm{mg} / \mathrm{L}$ ) of (a) MWCNTs, (b) GO, and (c) RGO for $24 \mathrm{~h}$ on the surface of the chorion of zebrafish embryos [Figure adapted from (Liu et al., 2014)].

After the exposure to the aforementioned nanomaterials', the heart rates of $48 \mathrm{hpf}$ embryonic zebrafish and $96 \mathrm{hpf}$ larval zebrafish were recorded. Besides, significant effects in the heart were observed in embryos treated with MWCNTs and GO, although RGO did not show any such effect.

Additionally, embryos treated with RGO were responsible for a dose-dependent decrease in the hatching rate. On the contrary, no effect on the hatching was observed in embryos treated with GO and MWCNTs. Similar results were shown in another experiment by different authors when two hpf zebrafish embryos were exposed to MWCNTs for $96 \mathrm{~h}$. Moreover, the experiment reported that the larva's body length and the heartbeats were decreased at $96 \mathrm{hpf}$, whereas no mortality or morphological abnormality was observed after exposure to MWCNTs. At present, extreme amounts of CNTs are not present in the environment. Therefore, along with focusing on high concentration exposure of CNTs, scientific studies must also emphasize its neurological and chronics effects. In case a situation such as reduced acetylcholinesterase (AchE) activity and a sixfold increase in brain levels of dopamine and serotonin were observed in the zebrafish exposed to $10 \mu \mathrm{L}$ dose of $30 \mathrm{mg} / \mathrm{kg}$ suspension of SWCNTs (da Rocha et al., 2019). An investigation reported that exposure of adult zebrafish to MWCNTs had not shown genotoxicity, but it was responsible for unwanted circumstances such as reversible inflammation in 
the gills (Filho et al., 2014). Another study by Girardi et al. demonstrated that higher concentrations (1ppm) of SWCNTs functionalized by polyethylene glycol led to toxic effects such as delayed hatching, decreased total larval length, and increased mortality in zebrafish (Girardi et al., 2017). Again, it was not responsible for genotoxicity, and no evidence validated the uptake of nanotubes by tissues. Pristine CNTs have shown higher biocompatibility, and even higher concentrations $(200 \mu \mathrm{g} / \mathrm{mL})$ of SWCNTs, MWCNTs, or carboxylated MW pristine CNTs have shown only a slight effect on embryo viability and development (Wang et al., 2016). Further studies using the zebrafish model system can expose the toxicity triggered due to carbon nanotubes along with the factors influencing it.

\section{Fullerenes}

Fullerenes $\left(\mathrm{C}_{60}\right)$ are the carbon-based nanomaterial widely known as Buckminster fullerenes or Bucky Balls and are composed of carbon atoms (Dizaj et al., 2015; Fako and Furgeson, 2009). These are the allotropes of carbon that were first discovered in 1985 and the present scenario; it is widely utilized in cosmetics, treatments for cloths, food supplements, electronics, fuel cells, and building materials (Kroto et al., 1985; Loutfy et al., 2002). Fullerenes also take a role in various biomedical applications like quenching of reactive oxygen species, drug and gene delivery, and bioimaging (Montellano et al., 2011; Partha and Conyers, 2009). Although the mass production of these compounds that are counted in ton quantities every year, they get stuck in the developmental stage (Robichaud et al., 2005). Henceforth, the rapid screening of these compounds can make a way to complete the developmental stage and get an adequate grip regarding their commercial utilization. The screening for toxicity may further assist in the reliable applications of carbon-based compounds like fullerenes. A study by Tegos et al. showed the antimicrobial property of fullerene against several bacteria such as Streptococcus spp., E. coli, and Salmonella (Tegos et al., 2005).

Assessments using the zebrafish model system also came up with conflicting results, which reported the toxic effects triggered due to fullerene. In an investigation by Usenko et al., 24 hpf embryonic zebrafish were incubated with fullerene $\mathrm{C}_{60}$ at $100-500 \mathrm{ppb}$, fullerenol $\mathrm{C}_{60}(\mathrm{OH})_{24}$ at $0.1-5 \mathrm{ppm}$, fullerene $\mathrm{C}_{70}$ at $20-500$ ppb (Figure 7) (Usenko et al., 2007).

The results described high mortality caused by five ppm $\mathrm{C}_{60}(\mathrm{OH})_{24}$, and malformation of the caudal fin, pericardial malformation, and $100 \%$ mortality rate was observed in the ones treated with $\mathrm{C}_{60}$ at $100-500 \mathrm{ppb}$ and $\mathrm{C}_{70}$ at $20-500 \mathrm{ppb}$. Even though $\mathrm{C} 60$ showed developmental toxicity, $\mathrm{C}_{60}(\mathrm{OH})_{24}$, and $\mathrm{C}_{70}$, the toxic effects of $\mathrm{C} 60(\mathrm{OH}) 24$ were less than $\mathrm{C}_{60}$ and $\mathrm{C}_{70}$. An extensive study by the same author showed the effects of light activation and antioxidants on developmental toxicity in zebrafish, directed by $\mathrm{C}_{60}$ (Usenko et al., 2008). The results indicated reduced developmental toxicity in the dark in comparison with the light condition. The co-exposure to the GSH synthesis inhibitor, buthionine sulfoximine (BSO), or diethylmaleate (DEM) were responsible for the elevated mortality rate.

In contrast, decreased mortality and pericardial edema were observed subsequently to coexposure to $\mathrm{C}_{60}$ and antioxidants, $\mathrm{N}$ - acetylcysteine (NAC), or ascorbic acid. Additionally, after the embryonic zebrafish were exposed to $\mathrm{C}_{60}$ at $200 \mathrm{ppb}$, the significant stress response genes were found to be upregulated. Moreover, the study concluded that the $\mathrm{C}_{60}$ might have acted as a pro-oxidant and have provoked toxicity through oxidative stress. Along with the light condition, several other factors influence fullerene's toxicity, and the charge properties are also one of them. Studies have suggested that apoptotic cell death can be avoided by the water-soluble fullerenes (Beuerle et al., 2007).

In an investigation of water-soluble fullerenes, zebrafish embryos were treated with positively-charged fullerenes and negativelycharged fullerenes of similar structures (Beuerle et al., 2007). The investigation also reported augmented toxicity induced by the positively charged fullerenes compared to negatively charged fullerenes. Whereas many considerable results, such as excellent biocompatibility accompanied by homogeneous distribution in larval zebrafish and little toxicity, were shown by the multi-shell fullerene structures known as nano-onions (D'Amora et al., 2016). Upcoming studies may lead to gain more described information concerning the toxicity and the biocompatibility of fullerenes. 
a

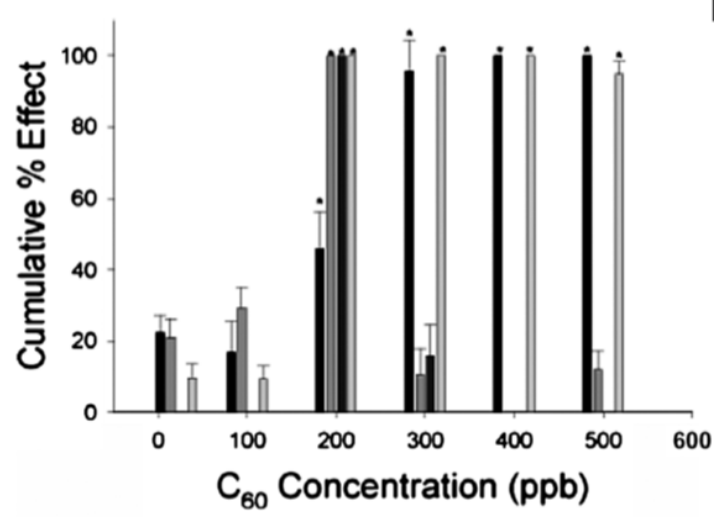

C

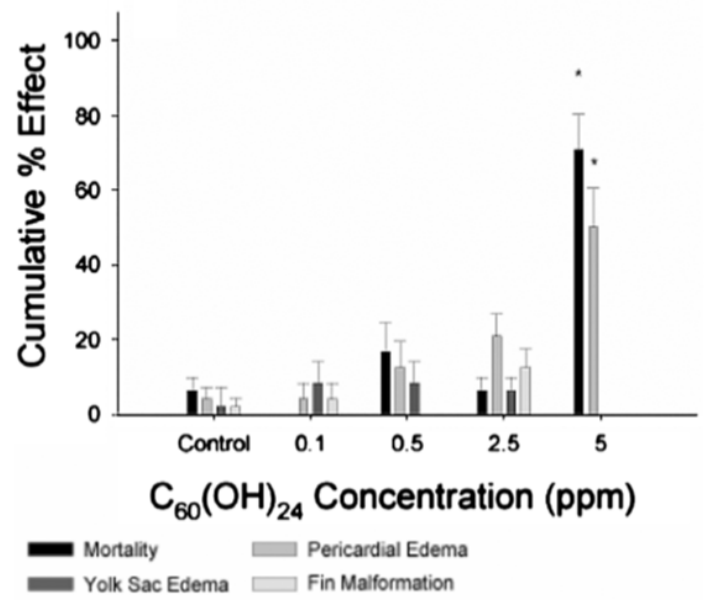

b
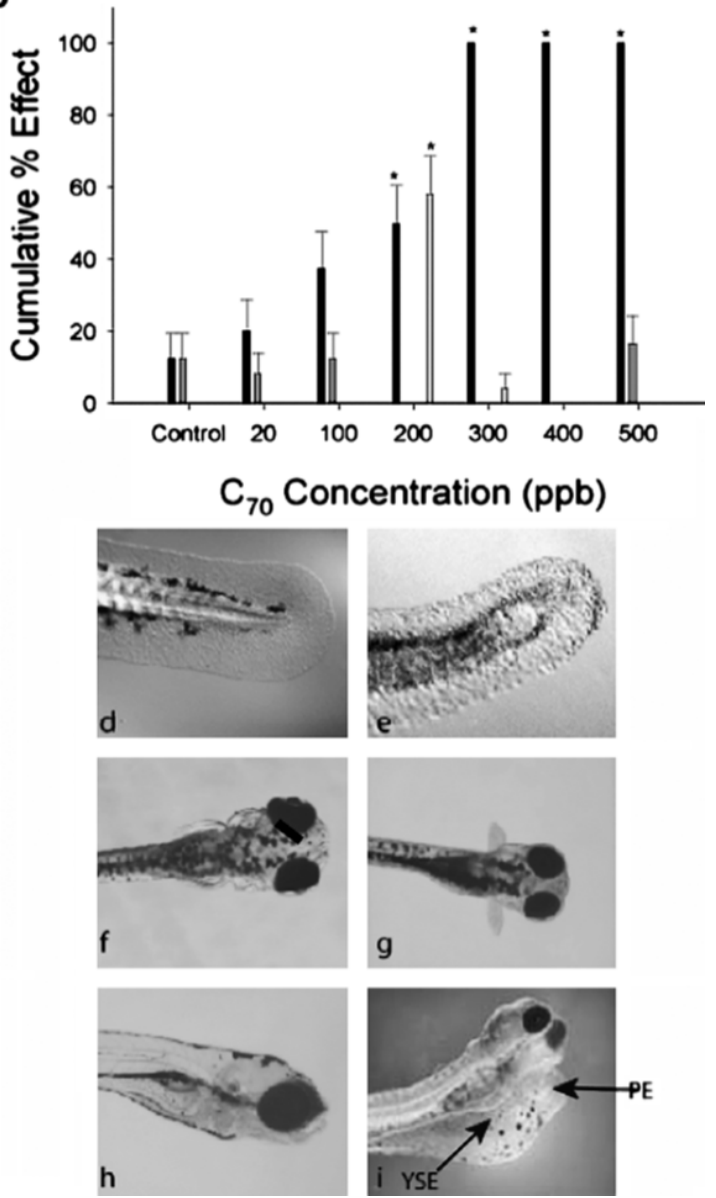

Figure 7. Concentration-responses observed for embryonic zebrafish exposed to (a) C60, (b) C70, and (c) C60(OH)24 from 24 to 96 hpf; evaluated daily until 6 dpf. Values represent the \% showing the effect by day 6 (cumulative \% effect): mortality, pericardial edema, yolk sac edema, and fin malformations. Representative images of the caudal fins for (d) control and (e) $200 \mathrm{ppb} C 60$-exposed animals are given. Representative images of the pectoral fin for $(f)$ control and ( $g$ ) $3500 \mathrm{ppb} C 60(\mathrm{OH}) 24-$ exposed animals. Representative images of (h) 1\% dimethyl sulfoxide (DMSO) control head at 6 dpf and (i) 200 ppb C60exposed head at 6 dpf; arrows designate pericardial edema (PE) and yolk sac edema (YSE). Significance was determined using Fisher's exact test $\left({ }^{*} p<0.05\right)$ compared to $1 \%$ DMSO control $(N=24)$ [Figure adapted from Usenko et al., 2007)].

\section{Zebrafish model for toxicity screening of Quantum dots (QDs)}

Semiconductor crystals known as quantum dots (QDs) have emerged to be an essential nanomaterial from the past few decades. The diameter of QDs ranges from 2-10 nm, and it comprises around 200-10,000 atoms that result in the formation of a significant class of fluorescent labels which is further utilized for biosensing, biomedical, and other biological applications (Libralato et al., 2017).

The excellent fluorescence properties possessed by QDs are due to their very small size and their exclusive photophysical and photochemical properties. Apart from that, QDs also show several other admirable properties: tunable emission spectra, high brightness, and specific, elevated bioactivity when coated and linked to functional groups. The two most frequently utilized QDs used for biological applications are cadmium selenide (CdSe) and cadmium telluride (CdTe). CdSe and CdTe are the nanocrystals with extremely bright fluorescent properties, which are considered powerful labeling agents for diverse in vivo applications (Haque and Ward, 2018a).

However, the awareness related to the health risks and the potential toxicity given rise by the exposure of these NPs is very little known, and most importantly, these NPs are exposed to the environment at a very large scale. Several experiments with the zebrafish model came up with results that reported endurable and modest toxicity when higher concentrations of CdSe QDs were directly injected in embryonic zebrafish (Rieger et al., 2005; Zhang et al., $2012 b$ ). On the contrary, an investigation by 
Tang et al. described the adverse toxic effects induced due to CdTe QDs in Zebrafish liver (ZFL) cells at an equal $\mathrm{Cd}^{2+}$ concentration (Tang et al., 2013). Nevertheless, the results showing the effects of free $\mathrm{Cd}^{2+}$ did not lead to adequate explanations regarding the toxicities of QDs in organisms. However, another study led to a range of behavioral and developmental disturbances when after zebrafish embryos were exposed to $\mathrm{Cd}$ Te QDs were exposed to embryonic zebrafish (Zhang et al., 2012a). Another form of QDs and the most commonly used ones are the graphene quantum dots (GQDs).

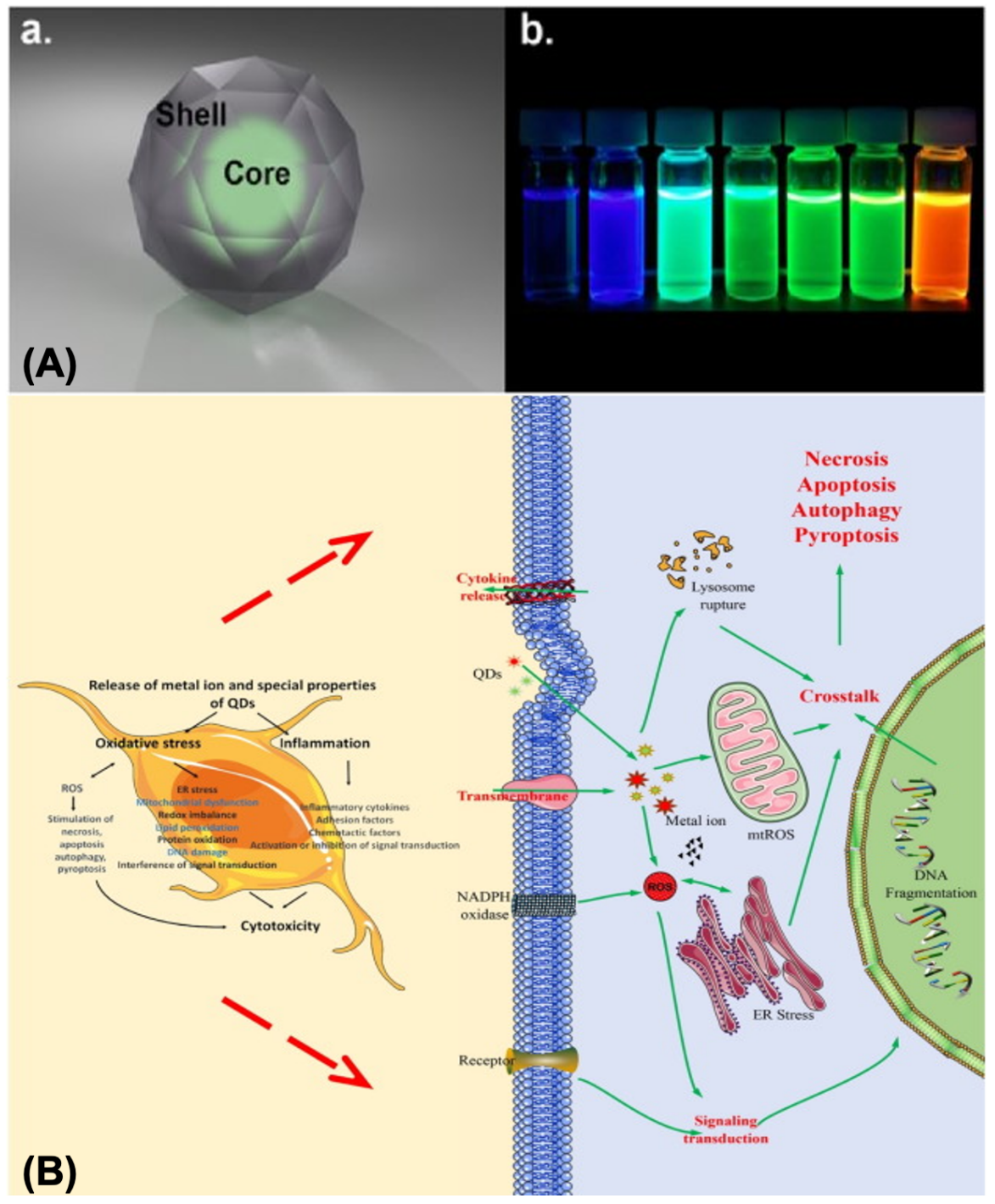

Figure 8. (A) The basic structure and fluorescence image of quantum dots (QDs). (a.) A typical QD is composed of core and shells. It also can be designed with addition of variable outer layer, like peptides, antibodies, oligonucleotides and so on for satisfying biocompatibility. (b.) Fluorescence image of QDs. In general, the fluorescence of QDs is dependent on the size. The larger QDs of the same material exhibit a smaller energy bandgap and thus a photoluminescence emission in the red, whereas smaller QDs fluoresce in the blue. (B) The mechanisms of QDs-induced cytotoxicity. ER: endoplasmic reticulum stress; mtROS: mitochondrial reactive oxygen species. [Figure adapted from Wang and Tang, 2018)].

The lower toxicity and higher biocompatibility of GQDs is attractive for cellular imaging and drug delivery (X. Wang et al., 2014). It was found that at a concentration lower than 2 $\mathrm{mg} / \mathrm{ml}$, the GQDs were readily excreted from the adult zebrafish without any significant effect on its growth. Additionally, the GQDs were found to be accumulated in the digestive system, although the muscle, blood, and additional tissue showed no obvious photoluminescence signal. Further studies may reveal the toxicity mechanism of quantum dots and verify their biocompatibility, which may help in the appropriate utilization and applications of QDs. 
Toxicological endpoints in the Danio rerio model

The Zebrafish Vertebrae Danio rerio model has emerged as the most promising model for high-throughput development toxicity testing. With a three-dimensional perspective, the Zebrafish model has the potential to shed light on the development of toxicity pathways, recognizing the foundation of human health risk assessment (Nishimura et al., 2016). Changes in normal phenotypes indicate abnormalities in their normal physiology, so zebrafish's behavioral aspect is considered an essential parameter in toxicological testing. Both larvae and adults share behavioral responses. Toxicological endpoints such as angular development, tail malformations, body heartbeat rate, pigmentation are taken into account when a toxicity assay is completed. When exposed to a light source around 24-30 hpf, photo motor response and virtual motor responses (VMR) are two pioneers of high throughput analysis that cause reflex (via hindbrain pathway) increasing activity level. The larva's movement's duration deflects after exposure to chemicals, and the velocity of movement falls under (VMR) (Horzmann and Freeman, 2018). Toxicological assays take five days to complete, with toxicological endpoints being monitored on a regular basis. To put hallmarks on the tests, different staining procedures were used. The Zebrafish model's strength is put to good use on a large scale for the progression of science and society's betterment.

Toxicity analysis of supramolecular complexes in Zebrafish model

Supramolecular chemistry has attracted a considered amount of attention from chemists, material scientists, and biologists, looking to exploit non-covalent interactions, including hydrogen-bonding interaction, electrostatic interaction, hydrophobic interaction, hydrophilic interaction, $\pi-\pi$ stacking interaction, and van-der Waals force, in the spontaneous assembly of supramolecular molecules from building blocks. Interactions between enzymes and substrates have inspired Host-guest interactions studied in supramolecular chemistry. Furthermore, most of these cells' interactions are based on non-valent bonds between two or more molecules. Embryogenity and teratogenicity are among the most performed toxicity assays of developmental toxicity. Many model organisms have been validated for toxicity profiling. These models are alienated between validated models wholeembryo culture test (WEC), embryonic stem cell test (EST), and miro mass test (MM), and those that are not presently authenticated (although have proven scientific validity) as in the case of zebrafish, frog embryo teratogenesis assay (FETAX), in silico models for predicting embryotoxicity, in vitro cellular models dissimilar from the EST method, and methods using fragments of embryos. So far, only three in vitro methods (MM, EST, and WEC) have been legalized by an international agency (ECVAM) to be utilized for testing the embryotoxicity potential of chemicals. Simultaneously, other models, such as FETAX and zebrafish, have also demonstrated their cogency on behalf of this determination. Methods established on the employment of embryos permit the specific malformation anticipated after exposure to the chemical to be determined. However, methods based on cellular systems are more appropriate to govern the mechanism underlying adverse observed effect and still display a wide field for improving their prediction capability (Pamies et al., 2011). Because of the many advantages inherent to the zebrafish model, it is an increasingly popular vertebrate model for assessing nutraceuticals' safety and toxicity (Bian and Pei, 2016). The property of drug nanoparticles to penetrate or permeate various biological barriers has been known to afford them greater therapeutic efficacy. However, this same property provides the nano-carriers access to the primary cell machinery and hence an opportunity to hamper the normal biological functioning. Also unforeseen are the toxicity challenges which may be presented by the cellular accumulation and long-term retention of these fascinating therapeutic systems. This concern regarding the toxicological potential of drug nanoparticles has increasingly surfaced over the last few years through worldwide research dedicated to this field of research. Along with the cellular cytotoxicity assays, a focus has also been placed on assays that can identify the abnormalities arising due to the interaction of nanoparticles with cellular DNA, either directly or indirectly via oxidative and inflammatory reactions (Patravale et al., 2012).

Toxicity is also an important consideration while working with NPs used for manufacturing and other applications since those can result 
in environmental exposure. Nevertheless, it is one of the major aspects to be kept in mind for a pharmaceutical industry due to its reliability with the patients' health and condition where safety is the utmost concern. Several different platforms are available to assess toxicity, ranging from in vitro cell culture assays to basic model organisms. Cell lines and simpler organisms are useful for cellular level toxicity and genotoxicity studies, whereas higher vertebrates are essential to detect complex physiological interactions. However, rodent models are high cost, have relatively slow and inaccessible embryo development, require substantial amounts of material for testing due to their relatively larger size, and are accompanied by ethical concerns about their size. Apart from rodents, other primate models have similar ethical issues, instead of an even greater extent. Therefore, small, low-cost, but sophisticated models are very attractive for evaluating in vivo nanotoxicity. In this context, the zebrafish serves as a compelling, efficient, and cost-effective alternative. Cell lines, culture methods, cytotoxicity assay (MTT test), toxicity and anti-angiogenic assays with transgenic zebrafish, acute toxicity testing, skin sensitization tests, repeated dose toxicity testing, mutagenicity testing, chronic oral toxicity testing, carcinogenicity testing, toxicokinetics, neurotoxicity, developmental toxicity, genetic toxicity of testing, are usually under the criteria of pharmacological screening. Another important agenda is the analysis of toxins. Acute toxicity testing is carried out to determine the effect of a single dose on a particular animal species (Ducharme et al., 2015). Generally, it is recommended that acute toxicity testing should be carried out with two different animal species. In acute toxicological testing, the investigational product is administered at different dose levels, and the effect is observed for two weeks.

\section{Challenges of zebrafish as an animal} model

While its unique properties and notable features, such as rapid development and short generation time, make it a very appealing model organism that can be easily cultured in a laboratory setting, it has many disadvantages compared to mammalian models (Meyers, 2018). Though advantageous for use as a model, the zebrafish's small size also poses some difficulties, such as embryo embedding and sectioning and the types of recording equipment to be used for testing and evaluation (Padilla and MacPhail, 2011). As a result, the larvae's size is a significant constraint when dealing with them. Non-clinical toxicological studies rely on lower effectiveness as a result of this limitation. Furthermore, harmful exposures, dose analysis, chemical delivery, and absorption, among other things, could not be thoroughly investigated due to the limited scale. Because of the drawbacks mentioned above, the methods for calculating plasma levels and ADME (excretion) data of embryos have not progressed much and are still in the early stages (Grech et al., 2019; Villacrez et al., 2018). The genome sequencing of this model organism has already been performed, and it was discovered that compared to the human reference genome, $70 \%$ of human genes have at least one zebrafish orthologue (Howe et al., 2013). When it came to mutation, the zebrafish fell short of the benefits offered by mouse model organisms. The gene of interest in mouse models can be easily mutated and inserted through homologous recombination. However, the Sleeping Beauty transposon method has made it easier to introduce transgenic sequences into zebrafish genomes, making it easier to use fluorescent proteins, Cre-recombinases, cell-death proteins, and other proteins to develop new approaches (Davidson, 2003; Kawakami, 2007; Kwan et al., 2007).

There are also limitations and questions about the traceability of toxic potencies and affected tissues. The in-water procedure, in which chemicals are solubilized in water and then administered to zebrafish embryos, is commonly used for dosing zebrafish embryos. This has two drawbacks (Cassar et al., 2020). For starters, since the embryos are fully submerged in the solution, zebrafish in-water dosing can expose them to different chemicals than mammalian routes. Also, the administration routes change from fishes to mammalians, and henceforth we cannot realistically transpose data collected in zebrafish in other models, including humans. Second, insoluble or poorly soluble chemicals do not reach the embryos by in-water dosing and must be injected directly into the fish to expose them to such chemicals adequately. This must be achieved in order to screen zebrafish embryos at high throughput. 
Concerning these limitations, this model organism may not be routinely used to assess genotoxicity, biodistribution, pharmacokinetics and pharmacodynamics, transcriptional profiling.

Studies correlating the toxicological response of NP/NM in zebrafish and rodents

Zebrafish (Danio rerio) has been an established vertebrate model for studying different developmental toxicities and disease model systems and is being increasingly used for both pre-clinical studies and toxicological applications due to a range of favorable traits. Zebrafish require relatively inexpensive housing, making them very cost-effective, smaller in size, lowering housing requirements and the amount of agent required for testing. Moreover, they exhibit a high fecundity rate, with a single female producing around 300 eggs, further underpinning their competence as a model. The zebrafish and human genomics share approximately $70 \%$ similarity. In addition to that, good conservation of major developmental and physiological processes, with key organ systems, such as digestive, nervous, and cardiovascular systems, is also maintained, similar to humans. This, in turn, largely underpins the extensive equivalence in response to pharmacological agents between the two species, with many zebrafish models mimicking human diseases both genetically and phenotypically (Haque and Ward, 2018b).

Rodents and rabbit toxicity testing has been standardized for assessing acute toxicity by the US government since the 1950s. However, excessive expenditure and time consumption led to a backlog in chemical testing. In addition, up to a thousand new chemicals are introduced to the market yearly. Therefore, the need for new screening methods to keep pace with the development of new chemicals and protect both human and environmental health came up. Zebrafish have emerged as a viable vertebrate organism for chemical risk testing with over 1940 papers published since the initial paper exploring the effect of zinc sulfate during different stages of zebrafish development in 1965. They are a cost-effective model and show high homology to mammalian species.

Along with the mentioned advantages, they also provide a whole animal model advantage over cell lines allowing for metabolism and systemic interactions to mimic the human body's processes. Because of its small size, zebrafish embryos or larvae have been used successfully in high throughput toxicity screening. The embryos are preferred as testing models to adult fish because it is anticipated that early life stages feel less pain and distress than adult ones. Thus, zebrafish early life stages have been proposed as an effective model for toxicity profiling, and test guidelines have been developed and validated using zebrafish embryos as model organisms (Figure 9).

The appropriate selection of zebrafish developmental stage and the most suitable zebrafish line is crucial (Figure 9b). In addition, the route of administration of nanomedicines, such as incubation in media or intravenous injection, strongly influences experimental results. Zebrafish husbandry and maintenance dictates many factors, such as water conductivity, $\mathrm{pH}$, feeding, and fish density. They should be considered and controlled to guarantee consistent experimental conditions. The complementary application of classical in vitro systems and the zebrafish model (Figure 9c) offers the possibility to screen the effects of varying nanomedicine formulations and physicochemical properties under complex biological conditions. This facilitates the selection of promising lead formulations for subsequent rodent in vivo studies. The comparison of the classical 2-D in vitro system (Figure 9d, bottom left), the more sophisticated 3-D one (Figure 9d, top left), and the zebrafish model (Figure 9d, middle right) offer the possibility to assess nanomedicine interactions with biological environments under complex biological conditions.

In the drug discovery pipeline, safety pharmacology is a significant issue. The availability of an optimized preclinical, in vivo screening platform increases the chance to identify potentially successful nanomedicine formulations prior to translation into rodent in vivo studies. Unfortunately, so far only a few studies have assessed the reproducibility of rodent and rabbit toxicity with zebrafish embryo testing (Ducharme et al., 2015).

The zebrafish has been proposed as a model that can bridge the gap in this field between cell assays that are cost-effective but low in data content. In rodent assays, efficiency is less, whereas data content is high.

Zebrafish has been widely established as an alternative vertebrate model to investigate NM- 
mediated inflammation and oxidative stress. Along with these, other invertebrate models like Drosophila melanogaster, Caenorhabditis elegans are also being used routinely as substitutions to rodents for the study of disease pathogenesis, chemical toxicity, and screening new drug candidates for efficacy and toxicity. However, invertebrates vary in many aspects of their physiology and anatomy compared with humans, thus limiting their ability to accurately model many aspects of human diseases (Johnston et al., 2018).

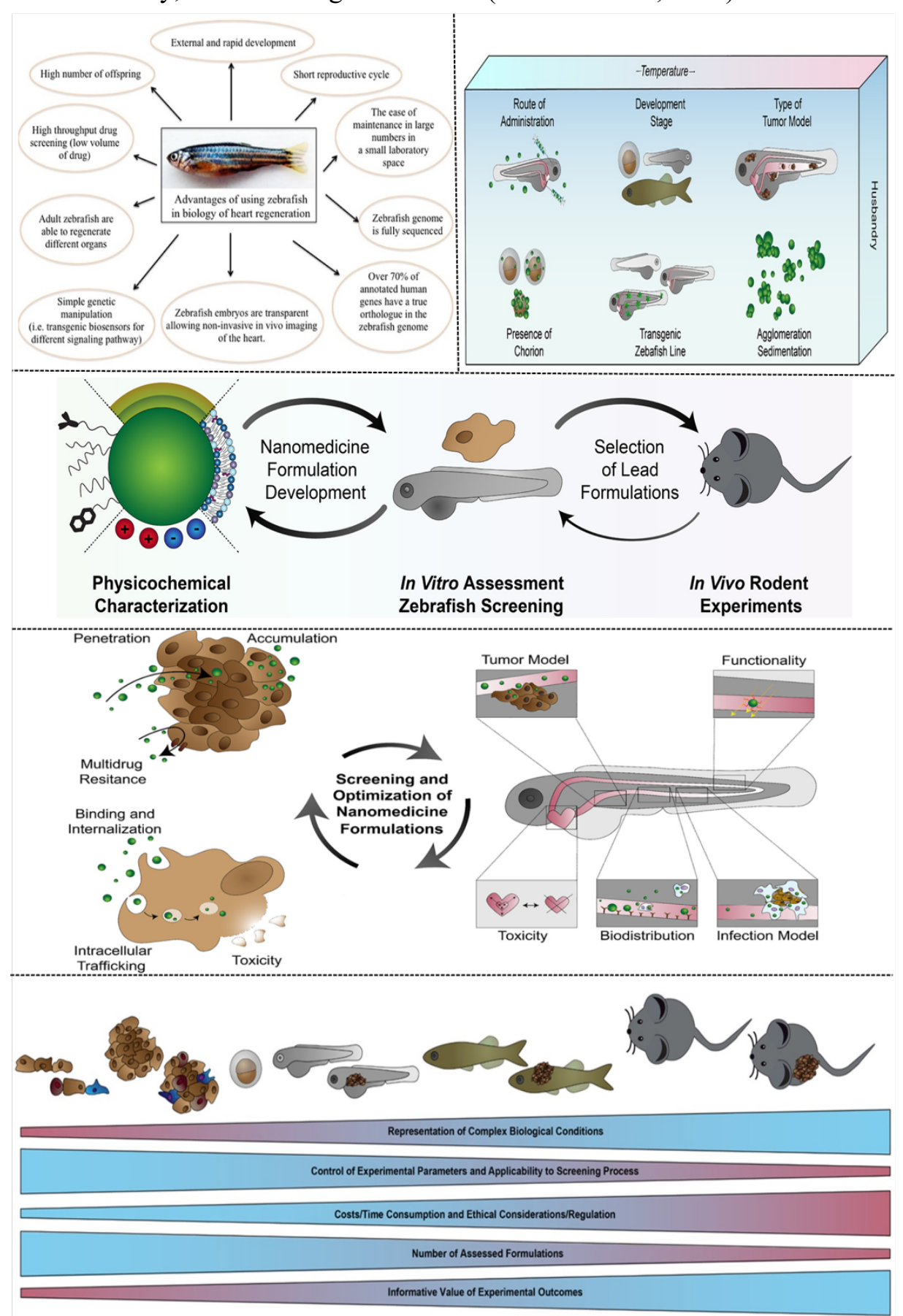

Fig 9. (a) The significance of using zebrafish as a model for toxicity profiling [Figure adapted from Beffagna, 2019)]. (b) Critical experimental parameters affecting the results of nanomedicine zebrafish studies. (c) Schematic representation of nanomedicine formulation design and optimization including the emerging zebrafish model. (d) Complementary application of in vitro and zebrafish model experimental set-ups for nanomedicine formulation design and optimization. (e) Comparison of different preclinical models for nanomedicine development. Factors affecting the appropriate selection of a model system during nanomedicine formulation design and optimization are represented. An optimized screening strategy relies on a smart combination of several available model systems and is key to success during clinical translation. (Figure $9 b, c, d$ and $e$ adapted from (Sieber et al., 2019). 


\section{Conclusion}

This review focuses on the several types of metal and metal oxide nanoparticles widely used in drug delivery approaches, such as fluorescent materials, bio-sensing devices, and catalysts in numerous chemical reaction processes. Among metal nanomaterials, gold, silver, copper, and aluminum, are predominantly used in environmental, pharmaceutical, and industrial applications. A variety of shapes and structures, from nanomembranes, nanofibers to nano scaffolds, can be fabricated from organic materials. Due to these several kinds of applications in therapeutics, tissue scaffolds, implantation technologies, nanomaterials have emerged to play a leading role in the biomedical field. For example, carbonbased nanomaterials, carbon nanotubes have gained mounting importance in biomedical scaffolds and implants. Other types of nanomaterials, such as carbon nanotubes, quantum dot-based nanomaterials, and fullerene molecules discussed in this study, are used in chemical reactions and pharmaceutical applications. Carbon nanotubes can be used as composite materials, implants, and drug delivery systems, while fullerene molecules can be used as lubricants. Overall, this review examines the advent of nanomaterials in today's environment from all angles and provides a compilation of data on their toxicity profiles focusing on zebrafish.

In light of the above studies, zebrafish has proved to be a promising and successful model organism for various toxicity screening and profiling studies. It is routinely used as an embryonic and larval model to perform in vitro experiments and developmental toxicity studies. Zebrafish may be used to regulate the toxicity of samples in early screening assays, often in a high-throughput manner. The Zebrafish embryo toxicity model is at the leading edge of toxicological research due to the short time required for analysis, short life cycle, high fertilization rate, transparent nature of embryos, and genetic data similarity. Their toxicity studies range from evaluating the toxicity of bioactive compounds or crude extracts from plants to determining the optimal process. Most of the studied extracts were polar, such as ethanol, methanol, and organic solvents, which were used to detect toxicity and bioactivity.

Compared to the other vertebrates, zebrafish have biological advantages, including high fecundity, external fertilization, optical transparency, and rapid development. In addition to that, zebrafish possess a highly developed immune system that is remarkably similar to that of humans. Therefore, it is anticipated that most signaling pathways and molecules involved in the immune response of mammals would also occur and act correspondingly in fish (Varela et al., 2017). Subsequently, the presence in fish of innate and adaptive immunity elements assists research in infectious processes, being susceptible to infectious by gram-negative and gram-positive bacteria, protozoa, viruses, fungi, and mycobacterium. Advancements in unique cloning, mutagenesis, and transgenesis techniques allowed identifying a significant number of mutants (Bailone et al., 2020).

\section{Conflict of Interests}

The authors declare no conflicts of interest. For a signed statement, please contact the journal office: editor@precisionnanomedicine.com

Quote this article as Verma SK, Nandi A, Sinha A, Patel P, Jha E, Mohanty S, Panda PK, Ahuja R, Mishra YK, Suar M, Zebrafish (Danio rerio) as an ecotoxicological model for Nanomaterial induced toxicity profiling, Precis. Nanomed. 2021;4(1):750-781; https://doi.org/10.33218/001c.21978

\section{References}

1. Adeleye, A.S., Oranu, E.A., Tao, M., Keller, A.A., 2016. Release and detection of nanosized copper from a commercial antifouling paint. Water Res. 102, 374-382. https://doi.org/10.1016/j.watres.2016.06.056

2. Ahamed, M., AlSalhi, M.S., Siddiqui, M.K.J., 2010. Silver nanoparticle applications and human health. Clin. Chim. Acta 411, 1841-1848. https://doi.org/10.1016/j.cca.2010.08.016

3. Akhtar, M.T., Ali, S., Rashidi, H., Van Der Kooy, F., Verpoorte, R., Richardson, M.K., 2013. Developmental effects of cannabinoids on zebrafish larvae. Zebrafish 10, 283-293. https://doi.org/10.1089/zeb.2012.0785 
4. Ali, S., Champagne, D.L., Spaink, H.P., Richardson, M.K., 2011. Zebrafish embryos and larvae: A new generation of disease models and drug screens. Birth Defects Res. Part C - Embryo Today Rev. 93, 115-133. https://doi.org/10.1002/bdrc.20206

5. Bai, C., Tang, M., 2020. Toxicological study of metal and metal oxide nanoparticles in zebrafish. J. Appl. Toxicol. 40, 37-63. https://doi.org/10.1002/jat.3910

6. Bai, W., Tian, W., Zhang, Z., He, X., Ma, Y., Liu, N., Chai, Z., 2010. Effects of copper nanoparticles on the development of zebrafish embryos. J. Nanosci. Nanotechnol. 10, 8670-8676. https://doi.org/10.1166/jnn.2010.2686

7. Bailone, R.L., Fukushima, H.C.S., Ventura Fernandes, B.H., De Aguiar, L.K., Corrêa, T., Janke, H., Grejo Setti, P., Roça, R.D.O., Borra, R.C., 2020. Zebrafish as an alternative animal model in human and animal vaccination research. Lab. Anim. Res. 36, 13. https://doi.org/10.1186/s42826020-00042-4

8. Bar-Ilan, O., Albrecht, R.M., Fako, V.E., Furgeson, D.Y., 2009. Toxicity assessments of multisized gold and silver nanoparticles in zebrafish embryos. Small 5, 1897-1910. https://doi.org/10.1002/smll.200801716

9. Basnet, R.M., Guarienti, M., Memo, M., 2017. Zebrafish Embryo as an In Vivo Model for Behavioral and Pharmacological Characterization of Methylxanthine Drugs. Int. J. Mol. Sci. . https://doi.org/10.3390/ijms18030596

10. Batley, G.E., Kirby, J., McLaughlin, M., 2011. Nanomaterials in Aquatic and Terrestrial Environments. Acc. Chem. Res. XXX. https://doi.org/10.4324/9780429331183

11. Beffagna, G., 2019. Zebrafish as a Smart Model to Understand Regeneration After Heart Injury: How Fish Could Help Humans. Front. Cardiovasc. Med. 6, 1-8. https://doi.org/10.3389/fcvm.2019.00107

12. Berman, J., Hsu, K., Look, A.T., 2003. Zebrafish as a model organism for blood diseases [WWW Document]. Br. J. Haematol. https://doi.org/10.1046/j.1365-2141.2003.04682.x

13. Beuerle, F., Witte, P., Hartnagel, U., Lebovitz, R., Parng, C., Hirsch, A., 2007. Cytoprotective activities of water-soluble fullerenes in zebrafish models. J. Exp. Nanosci. 2, 147-170. https://doi.org/10.1080/17458080701502091

14. Bian, W.-P., Pei, D.-S., 2016. Chapter 25 - Zebrafish Model for Safety and Toxicity Testing of Nutraceuticals, in: Gupta, R.C.B.T.-N. (Ed.), . Academic Press, Boston, pp. 333-339. https://doi.org/https://doi.org/10.1016/B978-0-12-802147-7.00025-5

15. Boran, H., Ulutas, G., 2016. Genotoxic effects and gene expression changes in larval zebrafish after exposure to $\mathrm{ZnCl} 2$ and $\mathrm{ZnO}$ nanoparticles. Dis. Aquat. Organ. 117, 205-214. https://doi.org/10.3354/dao02943

16. Brand, S.J., Botha, T.L., Wepener, V., 2020. Behavioural Response as a Reliable Measure of Acute Nanomaterial Toxicity in Zebrafish Larvae Exposed to a Carbon-Based Versus a Metal-Based Nanomaterial. African Zool. 55, 57-66. https://doi.org/10.1080/15627020.2019.1702098

17. Braunbeck, T., Lammer, E., 2006. Fish embryo toxicity assays. Contract 20, 725-731. https://doi.org/10.1007/s10811-007-9182-7

18. Browning, L.M., Lee, K.J., Huang, T., Nallathamby, P.D., Lowman, J.E., Nancy Xu, X.H., 2009. Random walk of single gold nanoparticles in zebrafish embryos leading to stochastic toxic effects on embryonic developments. Nanoscale 1, 138-152. https://doi.org/10.1039/b9nr00053d

19. Brun, N.R., Lenz, M., Wehrli, B., Fent, K., 2014. Comparative effects of zinc oxide nanoparticles and dissolved zinc on zebrafish embryos and eleuthero-embryos: Importance of zinc ions. Sci. Total Environ. 476-477, 657-666. https://doi.org/10.1016/j.scitotenv.2014.01.053

20. Brundo, M.V., Salvaggio, A., 2018. Zebrafish or Danio rerio: A New Model in Nanotoxicology Study. Recent Adv. Zebrafish Res. https://doi.org/10.5772/intechopen.74834

21. Busquet, F., Strecker, R., Rawlings, J.M., Belanger, S.E., Braunbeck, T., Carr, G.J., Cenijn, P., Fochtman, P., Gourmelon, A., Hübler, N., Kleensang, A., Knöbel, M., Kussatz, C., Legler, J., Lillicrap, A., Martínez-Jerónimo, F., Polleichtner, C., Rzodeczko, H., Salinas, E., Schneider, K.E., Scholz, S., van den Brandhof, E.J., van der Ven, L.T.M., Walter-Rohde, S., Weigt, S., Witters, H., Halder, M., 2014. OECD validation study to assess intra- and inter-laboratory reproducibility of the 
zebrafish embryo toxicity test for acute aquatic toxicity testing. Regul. Toxicol. Pharmacol. 69, 496-511. https://doi.org/10.1016/j.yrtph.2014.05.018

22. Caballero, M.V., Candiracci, M., 2018. Zebrafish as screening model for detecting toxicity and drugs efficacy. J. Unexplored Med. Data 3, 4. https://doi.org/10.20517/2572-8180.2017.15

23. Carpio, Y., Estrada, M.P., 2006. Zebrafish as a genetic model organism. Biotecnol. Apl. 23, 265270.

24. Cassar, S., Adatto, I., Freeman, J.L., Gamse, J.T., Iturria, I., Lawrence, C., Muriana, A., Peterson, R.T., Van Cruchten, S., Zon, L.I., 2020. Use of Zebrafish in Drug Discovery Toxicology. Chem. Res. Toxicol. 33, 95-118. https://doi.org/10.1021/acs.chemrestox.9b00335

25. Cha, C., Shin, S.R., Annabi, N., Dokmeci, M.R., 2013. Carbon-Based Nanomaterials: Multifunctional Materials for. ACS Nano 7, 2891-2897.

26. Chakraborty, C., Sarkar, B., Hsu, C.H., Wen, Z.H., Lin, C.S., Shieh, P.C., 2009. Future prospects of nanoparticles on brain targeted drug delivery. J. Neurooncol. 93, 285-286. https://doi.org/10.1007/s11060-008-9759-2

27. Chakraborty, C., Sharma, A.R., Sharma, G., Lee, S.S., 2016. Zebrafish :A complete animal model to enumerate the nanoparticle toxicity. J. Nanobiotechnol. 1-13. https://doi.org/10.1186/s12951016-0217-6

28. Chang, J., Ichihara, G., Shimada, Y., Tada-Oikawa, S., Kuroyanagi, J., Zhang, B., Suzuki, Y., Sehsah, R., Kato, M., Tanaka, T., Ichihara, S., 2015. Copper oxide nanoparticles reduce vasculogenesis in transgenic Zebrafish through down-regulation of vascular endothelial growth factor expression and induction of apoptosis. J. Nanosci. Nanotechnol. 15, 2140-2147. https://doi.org/10.1166/jnn.2015.9762

29. Chen, J., Dong, X., Xin, Y., Zhao, M., 2011. Effects of titanium dioxide nano-particles on growth and some histological parameters of zebrafish (Danio rerio) after a long-term exposure. Aquat. Toxicol. 101, 493-499. https://doi.org/10.1016/j.aquatox.2010.12.004

30. Chen, T.H., Lin, C.Y., Tseng, M.C., 2011. Behavioral effects of titanium dioxide nanoparticles on larval zebrafish (Danio rerio). Mar. Pollut. Bull. 63, 303-308. https://doi.org/10.1016/j.marpolbul.2011.04.017

31. Cheng, J., Flahaut, E., Shuk, H.C., 2007. Effect of carbon nanotubes on developing zebrafish (Danio rerio) embryos. Environ. Toxicol. Chem. 26, 708-716. https://doi.org/10.1897/06-272R.1

32. Choi, J.E., Kim, S., Ahn, J.H., Youn, P., Kang, J.S., Park, K., Yi, J., Ryu, D.Y., 2010. Induction of oxidative stress and apoptosis by silver nanoparticles in the liver of adult zebrafish. Aquat. Toxicol. 100, 151-159. https://doi.org/10.1016/j.aquatox.2009.12.012

33. D’Amora, M., Rodio, M., Bartelmess, J., Sancataldo, G., Brescia, R., Cella Zanacchi, F., Diaspro, A., Giordani, S., 2016. Biocompatibility and biodistribution of functionalized carbon nano-onions (f-CNOs) in a vertebrate model. Sci. Rep. 6, 1-9. https://doi.org/10.1038/srep33923

34. da Rocha, A.M., Kist, L.W., Almeida, E.A., Silva, D.G.H., Bonan, C.D., Altenhofen, S., Kaufmann, C.G.J., Bogo, M.R., Barros, D.M., Oliveira, S., Geraldo, V., Lacerda, R.G., Ferlauto, A.S., Ladeira, L.O., Monserrat, J.M., 2019. Neurotoxicity in zebrafish exposed to carbon nanotubes: Effects on neurotransmitters levels and antioxidant system. Comp. Biochem. Physiol. C. Toxicol. Pharmacol. 218, 30-35. https://doi.org/10.1016/j.cbpc.2018.12.008

35. Dankovich, T.A., Smith, J.A., 2014. Incorporation of copper nanoparticles into paper for point-ofuse water purification. Water Res. 63, 245-251. https://doi.org/10.1016/j.watres.2014.06.022

36. Das, S., Mitra, S., Khurana, S.M.P., Debnath, N., 2013. Nanomaterials for biomedical applications. Front. Life Sci. 7, 90-98. https://doi.org/10.1080/21553769.2013.869510

37. Davidson, A., 2003. Efficient gene delivery and gene expression in zebrafish using the Sleeping Beauty transposon. Dev. Biol. 263, 191-202. https://doi.org/10.1016/s0012-1606(03)00439-1

38. Dedeh, A., Ciutat, A., Treguer-Delapierre, M., Bourdineaud, J.P., 2015. Impact of gold nanoparticles on zebrafish exposed to a spiked sediment. Nanotoxicology 9, 71-80. https://doi.org/10.3109/17435390.2014.889238

39. Denluck, L., Wu, F., Crandon, L.E., Harper, B.J., Harper, S.L., 2018. Reactive oxygen species generation is likely a driver of copper based nanomaterial toxicity. Environ. Sci. Nano 5. https://doi.org/10.1039/c8en00055g 
40. Devi, G.P., Ahmed, K.B.A., Varsha, M.K.N.S., Shrijha, B.S., Lal, K.K.S., Anbazhagan, V., Thiagarajan, R., 2015. Sulfidation of silver nanoparticle reduces its toxicity in zebrafish. Aquat. Toxicol. 158, 149-156. https://doi.org/10.1016/j.aquatox.2014.11.007

41. Dizaj, S.M., Mennati, A., Jafari, S., Khezri, K., Adibkia, K., 2015. Antimicrobial activity of carbonbased nanoparticles. Adv. Pharm. Bull. 5, 19-23. https://doi.org/10.5681/apb.2015.003

42. Ducharme, N.A., Reif, D.M., Gustafsson, J.-A., Bondesson, M., 2015. Comparison of toxicity values across zebrafish early life stages and mammalian studies: Implications for chemical testing. Reprod. Toxicol. 55, 3-10. https://doi.org/https://doi.org/10.1016/j.reprotox.2014.09.005

43. Dumitrescu, E., Wallace, K., Andreescu, S., 2019. Nanotoxicity Assessment Using Embryonic Zebrafish. Methods Mol. Biol. 1894, 331-343. https://doi.org/10.1007/978-1-4939-8916-4_20

44. Eimon, P.M., Rubinstein, A.L., 2009. The use of in vivo zebrafish assays in drug toxicity screening. Expert Opin. Drug Metab. Toxicol. 5, 393-401. https://doi.org/10.1517/17425250902882128

45. Ema, M., Hougaard, K.S., Kishimoto, A., Honda, K., 2016. Reproductive and developmental toxicity of carbon-based nanomaterials: A literature review. Nanotoxicology 10, 391-412. https://doi.org/10.3109/17435390.2015.1073811

46. Embry, M.R., Belanger, S.E., Braunbeck, T.A., Galay-Burgos, M., Halder, M., Hinton, D.E., Léonard, M.A., Lillicrap, A., Norberg-King, T., Whale, G., 2010. The fish embryo toxicity test as an animal alternative method in hazard and risk assessment and scientific research. Aquat. Toxicol. 97, 79-87. https://doi.org/10.1016/j.aquatox.2009.12.008

47. Fako, V.E., Furgeson, D.Y., 2009. Zebrafish as a correlative and predictive model for assessing biomaterial nanotoxicity. Adv. Drug Deliv. Rev. 61, 478-486. https://doi.org/10.1016/j.addr.2009.03.008

48. Filho, J. de S., Matsubara, E.Y., Franchi, L.P., Martins, I.P., Rivera, L.M.R., Rosolen, J.M., Grisolia, C.K., 2014. Evaluation of carbon nanotubes network toxicity in zebrafish (danio rerio) model. Environ. Res. 134, 9-16. https://doi.org/10.1016/j.envres.2014.06.017

49. Ganesan, S., Anaimalai Thirumurthi, N., Raghunath, A., Vijayakumar, S., Perumal, E., 2016. Acute and sub-lethal exposure to copper oxide nanoparticles causes oxidative stress and teratogenicity in zebrafish embryos. J. Appl. Toxicol. 36, 554-567. https://doi.org/10.1002/jat.3224

50. Geffroy, B., Ladhar, C., Cambier, S., Treguer-Delapierre, M., Brèthes, D., Bourdineaud, J.P., 2012. Impact of dietary gold nanoparticles in zebrafish at very low contamination pressure: The role of size, concentration and exposure time. Nanotoxicology 6, 144-160. https://doi.org/10.3109/17435390.2011.562328

51. George, S., Lin, Sijie, Ji, Z., Thomas, C.R., Li, L., Mecklenburg, M., Meng, H., Wang, X., Zhang, H., Xia, T., Hohman, J.N., Lin, Shuo, Zink, J.I., Weiss, P.S., Nel, A.E., 2012. Surface defects on plate-shaped silver nanoparticles contribute to its hazard potential in a fish gill cell line and zebrafish embryos. ACS Nano 6, 3745-3759. https://doi.org/10.1021/nn204671v

52. Gerber, A., Bundschuh, M., Klingelhofer, D., Groneberg, D.A., 2013. Gold nanoparticles: Recent aspects for human toxicology. J. Occup. Med. Toxicol. 8, 1-6. https://doi.org/10.1186/1745-66738-32

53. Girardi, F.A., Bruch, G.E., Peixoto, C.S., Dal Bosco, L., Sahoo, S.K., Gonçalves, C.O.F., Santos, A.P., Furtado, C.A., Fantini, C., Barros, D.M., 2017. Toxicity of single-wall carbon nanotubes functionalized with polyethylene glycol in zebrafish (Danio rerio) embryos. J. Appl. Toxicol. 37, 214-221. https://doi.org/10.1002/jat.3346

54. Girigoswami, K., Viswanathan, M., Murugesan, R., Girigoswami, A., 2015. Studies on polymercoated zinc oxide nanoparticles: UV-blocking efficacy and in vivo toxicity. Mater. Sci. Eng. C 56, 501-510. https://doi.org/10.1016/j.msec.2015.07.017

55. Goodman, C.M., McCusker, C.D., Yilmaz, T., Rotello, V.M., 2004. Toxicity of gold nanoparticles functionalized with cationic and anionic side chains. Bioconjug. Chem. 15, 897-900. https://doi.org/10.1021/bc049951i

56. Grech, A., Tebby, C., Brochot, C., Bois, F.Y., Bado-Nilles, A., Dorne, J. Lou, Quignot, N., Beaudouin, R., 2019. Generic physiologically-based toxicokinetic modelling for fish: Integration of environmental factors and species variability. Sci. Total Environ. 651, 516-531. https://doi.org/10.1016/j.scitotenv.2018.09.163 
57. Griffitt, R.J., Weil, R., Hyndman, K.A., Denslow, N.D., Powers, K., Taylor, D., Barber, D.S., 2007. Exposure to copper nanoparticles causes gill injury and acute lethality in zebrafish (Danio rerio). Environ. Sci. Technol. 41, 8178-8186. https://doi.org/10.1021/es071235e

58. Haque, E., Ward, A.C., 2018a. Zebrafish as a model to evaluate nanoparticle toxicity. Nanomaterials 8, 1-18. https://doi.org/10.3390/nano8070561

59. Haque, E., Ward, A.C., 2018b. Zebrafish as a Model to Evaluate Nanoparticle Toxicity. Nanomater. . https://doi.org/10.3390/nano8070561

60. He, H., Pham-Huy, L.A., Dramou, P., Xiao, D., Zuo, P., Pham-Huy, C., 2013. Carbon nanotubes: Applications in pharmacy and medicine. Biomed Res. Int. 2013. https://doi.org/10.1155/2013/578290

61. Heilesen, L., 2017. Zebrafish is an important animal model [WWW Document]. 03.Mars. URL $\mathrm{http}: / / \mathrm{mbg}$. au.dk/en/news-and-events/news-item/artikel/the-zebrafish-is-an-important-animalmodel/ (accessed 8.23.20).

62. Hill, A.J., Teraoka, H., Heideman, W., Peterson, R.E., 2005. Zebrafish as a model vertebrate for investigating chemical toxicity. Toxicol. Sci. 86, 6-19. https://doi.org/10.1093/toxsci/kfi110

63. Horzmann, K.A., Freeman, J.L., 2018. Making waves: New developments in toxicology with the zebrafish. Toxicol. Sci. 163, 5-12. https://doi.org/10.1093/toxsci/kfy044

64. Horzmann, K.A., Freeman, J.L., 2016. Zebrafish get connected: Investigating neurotransmission targets and alterations in chemical toxicity. Toxics 4. https://doi.org/10.3390/toxics4030019

65. Hou, J., Wang, Xiangxue, Hayat, T., Wang, Xiangke, 2017. Ecotoxicological effects and mechanism of $\mathrm{CuO}$ nanoparticles to individual organisms. Environ. Pollut. 221, 209-217. https://doi.org/10.1016/j.envpol.2016.11.066

66. Howe, K., Clark, M.D., Torroja, C.F., Torrance, J., Berthelot, C., Muffato, M., Collins, J.E., Humphray, S., McLaren, K., Matthews, L., McLaren, S., Sealy, I., Caccamo, M., Churcher, C., Scott, C., Barrett, J.C., Koch, R., Rauch, G.J., White, S., Chow, W., Kilian, B., Quintais, L.T., Guerra-Assunção, J.A., Zhou, Y., Gu, Y., Yen, J., Vogel, J.H., Eyre, T., Redmond, S., Banerjee, R., Chi, J., Fu, B., Langley, E., Maguire, S.F., Laird, G.K., Lloyd, D., Kenyon, E., Donaldson, S., Sehra, H., Almeida-King, J., Loveland, J., Trevanion, S., Jones, M., Quail, M., Willey, D., Hunt, A., Burton, J., Sims, S., McLay, K., Plumb, B., Davis, J., Clee, C., Oliver, K., Clark, R., Riddle, C., Eliott, D., Threadgold, G., Harden, G., Ware, D., Mortimer, B., Kerry, G., Heath, P., Phillimore, B., Tracey, A., Corby, N., Dunn, M., Johnson, C., Wood, J., Clark, S., Pelan, S., Griffiths, G., Smith, M., Glithero, R., Howden, P., Barker, N., Stevens, C., Harley, J., Holt, K., Panagiotidis, G., Lovell, J., Beasley, H., Henderson, C., Gordon, D., Auger, K., Wright, D., Collins, J., Raisen, C., Dyer, L., Leung, K., Robertson, L., Ambridge, K., Leongamornlert, D., McGuire, S., Gilderthorp, R., Griffiths, C., Manthravadi, D., Nichol, S., Barker, G., Whitehead, S., Kay, M., Brown, J., Murnane, C., Gray, E., Humphries, M., Sycamore, N., Barker, D., Saunders, D., Wallis, J., Babbage, A., Hammond, S., Mashreghi-Mohammadi, M., Barr, L., Martin, S., Wray, P., Ellington, A., Matthews, N., Ellwood, M., Woodmansey, R., Clark, G., Cooper, J., Tromans, A., Grafham, D., Skuce, C., Pandian, R., Andrews, R., Harrison, E., Kimberley, A., Garnett, J., Fosker, N., Hall, R., Garner, P., Kelly, D., Bird, C., Palmer, S., Gehring, I., Berger, A., Dooley, C.M., Ersan-Ürün, Z., Eser, C., Geiger, H., Geisler, M., Karotki, L., Kirn, A., Konantz, J., Konantz, M., Oberländer, M., Rudolph-Geiger, S., Teucke, M., Osoegawa, K., Zhu, B., Rapp, A., Widaa, S., Langford, C., Yang, F., Carter, N.P., Harrow, J., Ning, Z., Herrero, J., Searle, S.M.J., Enright, A., Geisler, R., Plasterk, R.H.A., Lee, C., Westerfield, M., De Jong, P.J., Zon, L.I., Postlethwait, J.H., NüssleinVolhard, C., Hubbard, T.J.P., Crollius, H.R., Rogers, J., Stemple, D.L., 2013. The zebrafish reference genome sequence and its relationship to the human genome. Nature 496, 498-503. https://doi.org/10.1038/nature12111

67. Howe, K., Clark, M.D., Torroja, C.F., Torrance, J., Berthelot, C., Muffato, M., Collins, J.E., Humphray, S., Mclaren, K., Matthews, L., Mclaren, S., Sealy, I., Caccamo, M., White, S., Chow, W., Kilian, B., Churcher, C., Scott, C., Barrett, J.C., Koch, R., Zhou, Y., Gu, Y., Yen, J., Vogel, J., Eyre, T., Redmond, S., Banerjee, R., Chi, J., Fu, B., Langley, E., Maguire, S.F., Laird, G.K., Lloyd, D., Kenyon, E., Donaldson, S., Sehra, H., Almeida-king, J., Loveland, J., Trevanion, S., Jones, M., Quail, M., Willey, D., Hunt, A., Burton, J., Sims, S., Mclay, K., Plumb, B., Davis, J., Clee, C., 
Oliver, K., Clark, R., Riddle, C., Eliott, D., Threadgold, G., Harden, G., Ware, D., Mortimer, B., Kerry, G., Heath, P., Phillimore, B., Tracey, A., Corby, N., Dunn, M., Johnson, C., Wood, J., Clark, S., Pelan, S., Griffiths, G., Smith, M., Glithero, R., Howden, P., Barker, N., Stevens, C., Harley, J., Holt, K., Panagiotidis, G., Lovell, J., Beasley, H., Henderson, C., Gordon, D., Auger, K., Wright, D., Collins, J., Raisen, C., Dyer, L., Leung, K., Robertson, L., Ambridge, K., Leongamornlert, D., Mcguire, S., Gilderthorp, R., Griffiths, C., Manthravadi, D., Nichol, S., Barker, G., Whitehead, S., Kay, M., Brown, J., Murnane, C., Gray, E., Humphries, M., Sycamore, N., Barker, D., Saunders, D., Wallis, J., Babbage, A., Hammond, S., Mashreghi-mohammadi, M., Barr, L., Martin, S., Wray, P., Ellington, A., Matthews, N., Ellwood, M., Woodmansey, R., Clark, G., Cooper, J., Tromans, A., Grafham, D., Skuce, C., Pandian, R., Andrews, R., Harrison, E., Kimberley, A., Garnett, J., Fosker, N., Hall, R., Garner, P., Kelly, D., Bird, C., Palmer, S., Gehring, I., Berger, A., Herrero, J., Searle, S.M.J., Enright, A., Geisler, R., Plasterk, R.H.A., Lee, C., Westerfield, M., Rogers, J., Stemple, D.L., n.d. relationship to the human genome 1-6. https://doi.org/10.1038/nature12111

68. Hsu, C.-H., Wen, Z.-H., Lin, C.-S., Chakraborty, C., 2007. The Zebrafish Model: Use in Studying Cellular Mechanisms for a Spectrum of Clinical Disease Entities. Curr. Neurovasc. Res. 4, 111120. https://doi.org/10.2174/156720207780637234

69. Hu, Q., Guo, F., Zhao, F., Fu, Z., 2017. Effects of titanium dioxide nanoparticles exposure on parkinsonism in zebrafish larvae and PC12. Chemosphere 173, 373-379. https://doi.org/10.1016/j.chemosphere.2017.01.063

70. Hua, J., Vijver, M.G., Ahmad, F., Richardson, M.K., Peijnenburg, W.J.G.M., 2014a. Toxicity of different-sized copper nano- and submicron particles and their shed copper ions to zebrafish embryos. Environ. Toxicol. Chem. 33, 1774-1782. https://doi.org/10.1002/etc.2615

71. Hua, J., Vijver, M.G., Richardson, M.K., Ahmad, F., Peijnenburg, W.J.G.M., 2014b. Particlespecific toxic effects of differently shaped zinc oxide nanoparticles to zebrafish embryos (Danio rerio). Environ. Toxicol. Chem. 33, 2859-2868. https://doi.org/10.1002/etc.2758

72. Iijima, S., 1991. Helical microtubules of graphitic carbon. Nature 354, 56-58. https://doi.org/10.1038/354056a0

73. ISO - ISO/TS 19590:2017 - Nanotechnologies - Size distribution and concentration of inorganic nanoparticles in aqueous media via single particle inductively coupled plasma mass spectrometry [WWW Document], n.d. URL https://www.iso.org/standard/65419.html (accessed 7.18.20).

74. Jeevanandam, J., Chan, Y.S., Danquah, M.K., 2019. Zebrafish as a Model Organism to Study Nanomaterial Toxicity. Emerg. Sci. J. 3, 195-208. https://doi.org/10.28991/esj-2019-01182

75. Jin, S., Ye, K., 2007. Nanoparticle-mediated drug delivery and gene therapy. Biotechnol. Prog. 23, 32-41. https://doi.org/10.1021/bp060348j

76. Johnston, H.J., Verdon, R., Gillies, S., Brown, D.M., Fernandes, T.F., Henry, T.B., Rossi, A.G., Tran, L., Tucker, C., Tyler, C.R., Stone, V., 2018. Adoption of in vitro systems and zebrafish embryos as alternative models for reducing rodent use in assessments of immunological and oxidative stress responses to nanomaterials. Crit. Rev. Toxicol. 48, 252-271. https://doi.org/10.1080/10408444.2017.1404965

77. Jovanović, B., Ji, T., Palić, D., 2011. Gene expression of zebrafish embryos exposed to titanium dioxide nanoparticles and hydroxylated fullerenes. Ecotoxicol. Environ. Saf. 74, 1518-1525. https://doi.org/10.1016/j.ecoenv.2011.04.012

78. Kawakami, K., 2007. Tol2: A versatile gene transfer vector in vertebrates. Genome Biol. 8, 1-10. https://doi.org/10.1186/gb-2007-8-s1-s7

79. Kim, K.T., Zaikova, T., Hutchison, J.E., Tanguay, R.L., 2013. Gold nanoparticles disrupt zebrafish eye development and pigmentation. Toxicol. Sci. 133, 275-288. https://doi.org/10.1093/toxsci/kft081

80. Kim, S., Lee, S., Lee, I., 2012. Alteration of phytotoxicity and oxidant stress potential by metal oxide nanoparticles in Cucumis sativus. Water. Air. Soil Pollut. 223, 2799-2806. https://doi.org/10.1007/s1 1270-011-1067-3

81. Kotil, T., Akbulut, C., Yön, N.D., 2017. The effects of titanium dioxide nanoparticles on ultrastructure of zebrafish testis (Danio rerio). Micron 100, 38-44. https://doi.org/10.1016/j.micron.2017.04.006 
82. Kovrižnych, J.A., Sotńikóva, R., Zeljenková, D., Rollerová, E., Szabová, E., Wimmerov́a, S., 2013. Acute toxicity of 31 different nanoparticles to zebrafish (Danio rerio) tested in adulthood and in early life stages - Comparative study. Interdiscip. Toxicol. 6, 67-73. https://doi.org/10.2478/intox2013-0012

83. Kroto, H.W., Heath, J.R., O’Brien, S.C., Curl, R.F., Smalley, R.E., $1985 . \quad$ C60: Buckminsterfullerene. Nature 318, 162-163. https://doi.org/10.1038/318162a0

84. Kteeba, S.M., El-Adawi, H.I., El-Rayis, O.A., El-Ghobashy, A.E., Schuld, J.L., Svoboda, K.R., Guo, L., 2017. Zinc oxide nanoparticle toxicity in embryonic zebrafish: Mitigation with different natural organic matter. Environ. Pollut. 230, 1125-1140. https://doi.org/10.1016/j.envpol.2017.07.042

85. Kumari, P, Panda, PK., Jha, E, Kumari, K., Nisha, K, Mallick, MA., Verma, SK, 2017. Mechanistic insight to ROS and Apoptosis regulated cytotoxicity inferred by green synthesized $\mathrm{CuO}$ nanoparticles from Calotropis gigantea to Embryonic Zebrafish. Sci. Rep. 7, 1-17. https://doi.org/10.1038/s41598-017-16581-1

86. Kumari, S., kumari, P., Panda, P.K., Pramanik, N., Verma, S.K., Mallick, M.A., 2019. Molecular aspect of phytofabrication of gold nanoparticle from Andrographis peniculata photosystem II and their in vivo biological effect on embryonic zebrafish (Danio rerio). Environ. Nanotechnology, Monit. Manag. 11, 100201. https://doi.org/10.1016/j.enmm.2018.100201

87. Kwan, K.M., Fujimoto, E., Grabher, C., Mangum, B.D., Hardy, M.E., Campbell, D.S., Parant, J.M., Yost, H.J., Kanki, J.P., Chien, C. Bin, 2007. The Tol2kit: A multisite gateway-based construction Kit for Tol2 transposon transgenesis constructs. Dev. Dyn. 236, 3088-3099. https://doi.org/10.1002/dvdy.21343

88. Lammer, E., Carr, G.J., Wendler, K., Rawlings, J.M., Belanger, S.E., Braunbeck, T., 2009. Is the fish embryo toxicity test (FET) with the zebrafish (Danio rerio) a potential alternative for the fish acute toxicity test? Comp. Biochem. Physiol. - C Toxicol. Pharmacol. 149, 196-209. https://doi.org/10.1016/j.cbpc.2008.11.006

89. Lee, I.C., Ko, J.W., Park, S.H., Lim, J.O., Shin, I.S., Moon, C., Kim, S.H., Heo, J.D., Kim, J.C., 2016. Comparative toxicity and biodistribution of copper nanoparticles and cupric ions in rats. Int. J. Nanomedicine 11, 2883-2900. https://doi.org/10.2147/IJN.S106346

90. Lee, K.J., Browning, L.M., Nallathamby, P.D., Desai, T., Cherukuri, P.K., Xu, X.H.N., 2012. In vivo quantitative study of sized-dependent transport and toxicity of single silver nanoparticles using zebrafish embryos. Chem. Res. Toxicol. 25, 1029-1046. https://doi.org/10.1021/tx300021u

91. Lee, K.J., Browning, L.M., Nallathamby, P.D., Xu, X.H.N., 2013. Study of charge-dependent transport and toxicity of peptide-functionalized silver nanoparticles using zebrafish embryos and single nanoparticle plasmonic spectroscopy. Chem. Res. Toxicol. 26, 904-917. https://doi.org/10.1021/tx400087d

92. Legradi, J.B., Di Paolo, C., Kraak, M.H.S., van der Geest, H.G., Schymanski, E.L., Williams, A.J., Dingemans, M.M.L., Massei, R., Brack, W., Cousin, X., Begout, M.L., van der Oost, R., Carion, A., Suarez-Ulloa, V., Silvestre, F., Escher, B.I., Engwall, M., Nilén, G., Keiter, S.H., Pollet, D., Waldmann, P., Kienle, C., Werner, I., Haigis, A.C., Knapen, D., Vergauwen, L., Spehr, M., Schulz, W., Busch, W., Leuthold, D., Scholz, S., vom Berg, C.M., Basu, N., Murphy, C.A., Lampert, A., Kuckelkorn, J., Grummt, T., Hollert, H., 2018. An ecotoxicological view on neurotoxicity assessment. Environ. Sci. Eur. 30, 1-34. https://doi.org/10.1186/s12302-018-0173-x

93. Li, J., Ying, G.G., Jones, K.C., Martin, F.L., 2015. Real-world carbon nanoparticle exposures induce brain and gonadal alterations in zebrafish (Danio rerio) as determined by biospectroscopy techniques. Analyst 140, 2687-2695. https://doi.org/10.1039/c4an02227k

94. Li, Y.F., Chen, C., 2011. Fate and toxicity of metallic and metal-containing nanoparticles for biomedical applications. Small 7, 2965-2980. https://doi.org/10.1002/smll.201101059

95. Libralato, G., Galdiero, E., Falanga, A., Carotenuto, R., De Alteriis, E., Guida, M., 2017. Toxicity Effects of Functionalized Quantum Dots, Gold and Polystyrene Nanoparticles on Target Aquatic Biological Models: A Review. Molecules 22. https://doi.org/10.3390/molecules22091439

96. Liu, H., Wang, Xinxin, Wu, Y., Hou, J., Zhang, S., Zhou, N., Wang, Xiangke, 2019. Toxicity responses of different organs of zebrafish (Danio rerio) to silver nanoparticles with different particle 
sizes and surface coatings. Environ. Pollut. 246, 414-422. https://doi.org/10.1016/j.envpol.2018.12.034

97. Liu, X.T., Mu, X.Y., Wu, X.L., Meng, L.X., Guan, W.B., Ma, Y.Q., Sun, H., Wang, C.J., Li, X.F., 2014. Toxicity of multi-walled carbon nanotubes, graphene oxide, and reduced graphene oxide to zebrafish embryos. Biomed. Environ. Sci. 27, 676-683. https://doi.org/10.3967/bes2014.103

98. Liu, Z., Tabakman, S., Welsher, K., Dai, H., 2009. Carbon Nanotubes in Biology and Medicine: In vitro and in vivo Detection, Imaging and Drug Delivery. Nano Res. 2, 85-120. https://doi.org/10.1007/s12274-009-9009-8

99. Llorens, A., Lloret, E., Picouet, P.A., Trbojevich, R., Fernandez, A., 2012. Metallic-based micro and nanocomposites in food contact materials and active food packaging. Trends Food Sci. Technol. 24, 19-29. https://doi.org/10.1016/j.tifs.2011.10.001

100. Loutfy, R.O., Lowe, T.P., Moravsky, A.P., Katagiri, S., 2002. Commercial Production of Fullerenes and Carbon Nanotubes. Perspect. Fuller. Nanotechnol. 35-46. https://doi.org/10.1007/978-94-010-9598-3_4

101. McGrath, P., Li, C.Q., 2008. Zebrafish: a predictive model for assessing drug-induced toxicity. Drug Discov. Today 13, 394-401. https://doi.org/10.1016/j.drudis.2008.03.002

102. McNamara, K., Tofail, S.A.M., 2015. Nanosystems: The use of nanoalloys, metallic, bimetallic, and magnetic nanoparticles in biomedical applications. Phys. Chem. Chem. Phys. 17, 27981-27995. https://doi.org/10.1039/c5cp00831j

103. McNeil, P.L., Boyle, D., Henry, T.B., Handy, R.D., Sloman, K.A., 2014. Effects of metal nanoparticles on the lateral line system and behaviour in early life stages of zebrafish (Danio rerio). Aquat. Toxicol. 152, 318-323. https://doi.org/10.1016/j.aquatox.2014.04.022

104. Meyers, J.R., 2018. Zebrafish: Development of a Vertebrate Model Organism. Curr. Protoc. Essent. Lab. Tech. 16, 1-26. https://doi.org/10.1002/cpet.19

105. Meyyappan, M., 2005. Science and Applications Edited By, New York.

106. Mirzaei, H., Darroudi, M., 2017. Zinc oxide nanoparticles: Biological synthesis and biomedical applications. Ceram. Int. 43, 907-914. https://doi.org/10.1016/j.ceramint.2016.10.051

107. Mishra, Y.K., Adelung, R., 2018. ZnO tetrapod materials for functional applications. Mater. Today 21, 631-651. https://doi.org/10.1016/j.mattod.2017.11.003

108. Monteiro-Riviere, N.A., Tran, C.L., Tran, C.L., 2007. Nanotoxicology, Nanotoxicology. CRC Press. https://doi.org/10.3109/9781420045154

109. Montellano, A., Da Ros, T., Bianco, A., Prato, M., 2011. Fullerene C60 as a multifunctional system for drug and gene delivery. Nanoscale 3, 4035-4041. https://doi.org/10.1039/c1nr10783f

110. Nishimura, Y., Inoue, A., Sasagawa, S., Koiwa, J., Kawaguchi, K., Kawase, R., Maruyama, T., Kim, S., Tanaka, T., 2016. Using zebrafish in systems toxicology for developmental toxicity testing. Congenit. Anom. (Kyoto). 56, 18-27. https://doi.org/10.1111/cga.12142

111. Noman, M.T., Ashraf, M.A., Ali, A., 2019. Synthesis and applications of nano-TiO2: a review. Environ. Sci. Pollut. Res. 26, 3262-3291. https://doi.org/10.1007/s11356-018-3884-z

112. OECD/OCDE 2, n.d.

113. OECD GUIDELINE FOR TESTING OF CHEMICALS 2 Draft revised version, 2018.

114. Ong, K.J., Zhao, X., Thistle, M.E., Maccormack, T.J., Clark, R.J., Ma, G., Martinez-Rubi, Y., Simard, B., Loo, J.S.C., Veinot, J.G.C., Goss, G.G., 2014. Mechanistic insights into the effect of $\begin{array}{lllll}\text { nanoparticles on zebrafish hatch. } & \text { 295-304. }\end{array}$ https://doi.org/10.3109/17435390.2013.778345

115. organic-and-inorganic-nanoparticles [WWW Document], n.d. URL https://www.nanoshel.com/organic-and-inorganic-nanoparticles (accessed 7.27.20).

116. Padilla, S., MacPhail, R., 2011. Using Zebrafish to Assess Developmental Neurotoxicity, Reproductive and Developmental Toxicology. Elsevier Inc. https://doi.org/10.1016/B978-0-12382032-7.10015-3

117. Palaniappan, P.R., Pramod, K.S., 2011. The effect of titanium dioxide on the biochemical constituents of the brain of Zebrafish (Danio rerio): An FT-IR study. Spectrochim. Acta - Part A Mol. Biomol. Spectrosc. 79, 206-212. https://doi.org/10.1016/j.saa.2011.02.038 
118. Pamies, D., Estevan, C., Vilanova, E., Sogorb, M.A., 2017. Validated and nonvalidated mechanism-based methods for testing developmental toxicity, Reproductive and Developmental Toxicology. Elsevier Inc. https://doi.org/10.1016/B978-0-12-804239-7.00011-1

119. Pamies, D., Martínez, C.E., Sogorb, M.A., Vilanova, E., 2011. Chapter 11 - Mechanism-based models in reproductive and developmental toxicology, in: Gupta, R.C.B.T.-R. and D.T. (Ed.), . Academic Press, San Diego, pp. 135-146. https://doi.org/https://doi.org/10.1016/B978-0-12382032-7.10011-6

120. Partha, R., Conyers, J.L., 2009. Biomedical applications of functionalized fullerene-based nanomaterials. Int. J. Nanomedicine 4, 261-275. https://doi.org/10.2147/ijn.s5964

121. Patel, P., Kumari, P., K. Verma, S., Mallick, M.A., 2019. Cellular and Molecular Impact of Green Synthesized Silver Nanoparticles. Silver Nanoparticles - Heal. Saf. [Working Title] 1-15. https://doi.org/10.5772/intechopen.90717

122. Patravale, V., Dandekar, P., Jain, R., 2012. 4 - Nanotoxicology: evaluating toxicity potential of drug-nanoparticles, in: Patravale, V., Dandekar, P., Jain, R.B.T.-N.D.D. (Eds.), Woodhead Publishing Series in Biomedicine. Woodhead Publishing, pp. 123-155. https://doi.org/https://doi.org/10.1533/9781908818195.123

123. Pecoraro, R., D’Angelo, D., Filice, S., Scalese, S., Capparucci, F., Marino, F., Iaria, C., Guerriero, G., Tibullo, D., Scalisi, E.M., Salvaggio, A., Nicotera, I., Brundo, M. V., 2018. Toxicity evaluation of graphene oxide and titania loaded nafion membranes in zebrafish. Front. Physiol. 8, 1-7. https://doi.org/10.3389/fphys.2017.01039

124. Pecoraro, R., Marino, F., Salvaggio, A., Capparucci, F., Caro, G. Di, Iaria, C., Salvo, A., Rotondo, A., Tibullo, D., Guerriero, G., Scalisi, E.M., Zimbone, M., Impellizzeri, G., Brundo, M. V., 2017. Evaluation of chronic nanosilver toxicity to adult zebrafish. Front. Physiol. 8, 1-9. https://doi.org/10.3389/fphys.2017.01011

125. Powers, C.M., Slotkin, T.A., Seidler, F.J., Badireddy, A.R., Padilla, S., 2011. Silver nanoparticles alter zebrafish development and larval behavior: Distinct roles for particle size, coating and composition. Neurotoxicol. Teratol. 33, 708-714. https://doi.org/10.1016/j.ntt.2011.02.002

126. Ramsden, C.S., Henry, T.B., Handy, R.D., 2013. Sub-lethal effects of titanium dioxide nanoparticles on the physiology and reproduction of zebrafish. Aquat. Toxicol. 126, 404-413. https://doi.org/10.1016/j.aquatox.2012.08.021

127. Ramsden, C.S., Smith, T.J., Shaw, B.J., Handy, R.D., 2009. Dietary exposure to titanium dioxide nanoparticles in rainbow trout, (Oncorhynchus mykiss): No effect on growth, but subtle biochemical disturbances in the brain. Ecotoxicology 18, 939-951. https://doi.org/10.1007/s10646009-0357-7

128. Rani, R., Kumar, V., Rizzolio, F., 2018. Fluorescent Carbon Nanoparticles in Medicine for Cancer Therapy: An Update. ACS Med. Chem. Lett. 9, 5-6. https://doi.org/10.1021/acsmedchemlett.7b00523

129. Rieger, S., Kulkarni, R.P., Darcy, D., Fraser, S.E., Köster, R.W., 2005. Quantum dots are powerful multipurpose vital labeling agents in zebrafish embryos. Dev. Dyn. 234, 670-681. https://doi.org/10.1002/dvdy.20524

130. Robichaud, C.O., Tanzie, D., Weilenmann, U., Wiesner, M.R., 2005. Relative risk analysis of several manufactured nanomaterials: An insurance industry context. Environ. Sci. Technol. 39, 8985-8994. https://doi.org/10.1021/es0506509

131. Rocco, L., Santonastaso, M., Mottola, F., Costagliola, D., Suero, T., Pacifico, S., Stingo, V., 2015. Genotoxicity assessment of $\mathrm{TiO} 2$ nanoparticles in the teleost Danio rerio. Ecotoxicol. Environ. Saf. 113, 223-230. https://doi.org/10.1016/j.ecoenv.2014.12.012

132. Sangabathuni, S., Murthy, R.V., Chaudhary, P.M., Subramani, B., Toraskar, S., Kikkeri, R., 2017. Mapping the Glyco-Gold Nanoparticles of Different Shapes Toxicity, Biodistribution and Sequestration in Adult Zebrafish. Sci. Rep. 7, 1-7. https://doi.org/10.1038/s41598-017-03350-3

133. Sieber, S., Grossen, P., Bussmann, J., Campbell, F., Kros, A., Witzigmann, D., Huwyler, J., 2019. Zebrafish as a preclinical in vivo screening model for nanomedicines. Adv. Drug Deliv. Rev. 151-152, 152-168. https://doi.org/10.1016/j.addr.2019.01.001 
134. Silva, S., Almeida, A.J., Vale, N., 2019. Combination of cell-penetrating peptides with nanoparticles for therapeutic application: A review. Biomolecules 9. https://doi.org/10.3390/biom9010022

135. Song, L., Vijver, M.G., Peijnenburg, W.J.G.M., Galloway, T.S., Tyler, C.R., 2015. A comparative analysis on the in vivo toxicity of copper nanoparticles in three species of freshwater fish. Chemosphere 139, 181-189. https://doi.org/10.1016/j.chemosphere.2015.06.021

136. Sousa Borges, R., César Matias Pereira, A., Custodio de Souza, G., Carlos Tavares Carvalho, J., 2020. Histopathology of Zebrafish ( Danio rerio ) in Nonclinical Toxicological Studies of New Drugs . Zebrafish Biomed. Res. https://doi.org/10.5772/intechopen.88639

137. Srinivasan, M., Venkatesan, M., Arumugam, V., Natesan, G., Saravanan, N., Murugesan, S., Ramachandran, S., Ayyasamy, R., Pugazhendhi, A., 2019. Green synthesis and characterization of titanium dioxide nanoparticles (TiO 2 NPs) using Sesbania grandiflora and evaluation of toxicity in zebrafish embryos. Process Biochem. 80, 197-202. https://doi.org/10.1016/j.procbio.2019.02.010

138. Subhapriya, S., Gomathipriya, P., 2018. Green synthesis of titanium dioxide (TiO2) nanoparticles by Trigonella foenum-graecum extract and its antimicrobial properties. Microb. Pathog. 116, 215-220. https://doi.org/10.1016/j.micpath.2018.01.027

139. Sun, Y., Zhang, G., He, Z., Wang, Y., Cui, J., Li, Y., 2016. Effects of copper oxide nanoparticles on developing zebrafish embryos and larvae. Int. J. Nanomedicine 11, 905-918. https://doi.org/10.2147/IJN.S100350

140. Tamilvanan, A., Balamurugan, K., Ponappa, K., Kumar, B.M., 2014. Copper nanoparticles: Synthetic strategies, properties and multifunctional application. Int. J. Nanosci. 13. https://doi.org/10.1142/S0219581X14300016

141. Tang, S., Cai, Q., Chibli, H., Allagadda, V., Nadeau, J.L., Mayer, G.D., 2013. Cadmium sulfate and CdTe-quantum dots alter DNA repair in zebrafish (Danio rerio) liver cells. Toxicol. Appl. Pharmacol. 272, 443-452. https://doi.org/10.1016/j.taap.2013.06.004

142. Tanguay, R.L., Harper, S.L., Carriere, J.L., Miller, J.M., Hutchison, J.E., Maddux, B.L.S., 2011. Systematic Evaluation of Nanomaterial Toxicity: Utility of Standardized Materials and Rapid Assays. ACS Nano 5, 4688-4697.

143. Tegos, G.P., Demidova, T.N., Arcila-Lopez, D., Lee, H., Wharton, T., Gali, H., Hamblin, M.R., 2005. Cationic fullerenes are effective and selective antimicrobial photosensitizers. Chem. Biol. 12, 1127-1135. https://doi.org/10.1016/j.chembiol.2005.08.014

144. Truong, L., Zaikova, T., Richman, E.K., Hutchison, J.E., Tanguay, R.L., 2012. Media ionic strength impacts embryonic responses to engineered nanoparticle exposure. Nanotoxicology 6, 691-699. https://doi.org/10.3109/17435390.2011.604440

145. Usenko, C.Y., Harper, S.L., Tanguay, R.L., 2008. Fullerene C60 exposure elicits an oxidative stress response in embryonic zebrafish. Toxicol. Appl. Pharmacol. 229, 44-55. https://doi.org/10.1016/j.taap.2007.12.030

146. Usenko, C.Y., Harper, S.L., Tanguay, R.L., 2007. In vivo evaluation of carbon fullerene toxicity using embryonic zebrafish. Carbon N. Y. 45, 1891-1898. https://doi.org/10.1016/j.carbon.2007.04.021

147. Varela, M., Figueras, A., Novoa, B., 2017. Modelling viral infections using zebrafish: Innate immune response and antiviral research. Antiviral Res. 139, 59-68. https://doi.org/https://doi.org/10.1016/j.antiviral.2016.12.013

148. Verma, S.K., Jha, E., Panda, P.K., Mishra, A., Thirumurugan, A., Das, B., Parashar, S.K.S., Suar, M., 2018a. Rapid novel facile biosynthesized silver nanoparticles from bacterial release induce biogenicity and concentration dependent in vivo cytotoxicity with embryonic Zebrafish-A mechanistic insight. Toxicol. Sci. 161, 125-138. https://doi.org/10.1093/toxsci/kfx204

149. Verma, S.K., Jha, E., Panda, P.K., Mukherjee, M., Thirumurugan, A., Makkar, H., Das, B., Parashar, S.K.S., Suar, M., 2018b. Mechanistic insight into ROS and neutral lipid alteration induced toxicity in the human model with fins (Danio rerio) by industrially synthesized titanium dioxide nanoparticles. Toxicol. Res. (Camb). 7, 244-257. https://doi.org/10.1039/c7tx00300e 
150. Verma, S.K., Jha, E., Panda, P.K., Thirumurugan, A., Suar, M., 2019. Biological Effects of Green-Synthesized Metal Nanoparticles: A Mechanistic View of Antibacterial Activity and Cytotoxicity 145-171. https://doi.org/10.1007/978-3-030-04477-0_6

151. Verma, S.K., Panda, P.K., Jha, E., Suar, M., Parashar, S.K.S., 2017. Altered physiochemical properties in industrially synthesized $\mathrm{ZnO}$ nanoparticles regulate oxidative stress; Induce in vivo cytotoxicity in embryonic zebrafish by apoptosis. Sci. Rep. 7, 1-16. https://doi.org/10.1038/s41598-017-14039-y

152. Villacrez, M., Hellman, K., Ono, T., Sugihara, Y., Rezeli, M., Ek, F., Marko-Varga, G., Olsson, R., 2018. Evaluation of Drug Exposure and Metabolism in Locust and Zebrafish Brains Using Mass Spectrometry Imaging. ACS Chem. Neurosci. 9, 1994-2000. https://doi.org/10.1021/acschemneuro.7b00459

153. Wang, J., Zhu, X., Zhang, X., Zhao, Z., Liu, H., George, R., Wilson-Rawls, J., Chang, Y., Chen, Y., 2011. Disruption of zebrafish (Danio rerio) reproduction upon chronic exposure to $\mathrm{TiO} 2$ nanoparticles. Chemosphere 83, 461-467. https://doi.org/10.1016/j.chemosphere.2010.12.069

154. Wang, R., Meredith, A.N., Lee, M., Deutsch, D., Miadzvedskaya, L., Braun, E., Pantano, P., Harper, S., Draper, R., 2016. Toxicity assessment and bioaccumulation in zebrafish embryos exposed to carbon nanotubes suspended in Pluronic ${ }^{\circledR}$ F-108. Nanotoxicology 10, 689-698. https://doi.org/10.3109/17435390.2015.1107147

155. Wang, X., Sun, X., Lao, J., He, H., Cheng, T., Wang, M., Wang, S., Huang, F., 2014. Multifunctional graphene quantum dots for simultaneous targeted cellular imaging and drug delivery. Colloids Surfaces B Biointerfaces 122, 638-644. https://doi.org/10.1016/j.colsurfb.2014.07.043

156. Wang, Y., Tang, M., 2018. Review of in vitro toxicological research of quantum dot and potentially involved mechanisms. Sci. Total Environ. 625, 940-962. https://doi.org/10.1016/j.scitotenv.2017.12.334

157. Wang, Y.J., He, Z.Z., Fang, Y.W., Xu, Y., Chen, Y.N., Wang, G.Q., Yang, Y.Q., Yang, Z., Li, Y.H., 2014. Effect of titanium dioxide nanoparticles on zebrafish embryos and developing retina. Int. J. Ophthalmol. 7, 917-923. https://doi.org/10.3980/j.issn.2222-3959.2014.06.01

158. Xin, Q., Rotchell, J.M., Cheng, J., Yi, J., Zhang, Q., 2015. Silver nanoparticles affect the neural development of zebrafish embryos. J. Appl. Toxicol. 35, 1481-1492. https://doi.org/10.1002/jat.3164

159. Xiong, D., Fang, T., Yu, L., Sima, X., Zhu, W., 2011. Effects of nano-scale TiO2, ZnO and their bulk counterparts on zebrafish: Acute toxicity, oxidative stress and oxidative damage. Sci. Total Environ. 409, 1444-1452. https://doi.org/10.1016/j.scitotenv.2011.01.015

160. Xu, J., Zhang, Q., Li, X., Zhan, S., Wang, L., Chen, D., 2017. The effects of copper oxide nanoparticles on dorsoventral patterning, convergent extension, and neural and cardiac development of zebrafish. Aquat. Toxicol. 188, 130-137. https://doi.org/10.1016/j.aquatox.2017.05.002

161. Xu, J.P., Zhang, R.T., Zhang, T., Zhao, G., Huang, Y., Wang, H.L., Liu, J.X., 2017. Copper impairs zebrafish swimbladder development by down-regulating Wnt signaling. Aquat. Toxicol. 192, 155-164. https://doi.org/10.1016/j.aquatox.2017.09.018

162. Yeh, Y.C., Creran, B., Rotello, V.M., 2012. Gold nanoparticles: Preparation, properties, and applications in bionanotechnology. Nanoscale 4, 1871-1880. https://doi.org/10.1039/c1nr11188d

163. Zhang, W., Lin, K., Miao, Y., Dong, Q., Huang, C., Wang, H., Guo, M., Cui, X., 2012a. Toxicity assessment of zebrafish following exposure to CdTe QDs. J. Hazard. Mater. 213-214, 413-420. https://doi.org/10.1016/j.jhazmat.2012.02.014

164. Zhang, W., Sun, X., Chen, L., Lin, K.F., Dong, Q.X., Huang, C.J., Fu, R.B., Zhu, J., 2012b. Toxicological effect of joint cadmium selenium quantum dots and copper ion exposure on zebrafish. Environ. Toxicol. Chem. 31, 2117-2123. https://doi.org/10.1002/etc.1918

165. Zhao, X., Wang, S., Wu, Y., You, H., Lv, L., 2013. Acute ZnO nanoparticles exposure induces developmental toxicity, oxidative stress and DNA damage in embryo-larval zebrafish. Aquat. Toxicol. 136-137, 49-59. https://doi.org/10.1016/j.aquatox.2013.03.019 
166. Zhu, X., Zhu, L., Duan, Z., Qi, R., Li, Y., Lang, Y., 2008. Comparative toxicity of several metal oxide nanoparticle aqueous suspensions to Zebrafish (Danio rerio) early developmental stage. J. Environ. Sci. Heal. - Part A Toxic/Hazardous Subst. Environ. Eng. 43, 278-284. https://doi.org/10.1080/10934520701792779 